\title{
Extension of Tensor-Product Generalized and Dense-Norm Summation-by-Parts Operators to Curvilinear Coordinates
}

DOI:

10.1007/s10915-019-01011-3

\section{Document Version}

Accepted author manuscript

Link to publication record in Manchester Research Explorer

\section{Citation for published version (APA):}

Del Rey Fernández, D. C., Boom, P. D., Carpenter, M. H., \& Zingg, D. W. (2019). Extension of Tensor-Product Generalized and Dense-Norm Summation-by-Parts Operators to Curvilinear Coordinates. Journal of Scientific Computing, 80(3), 1957-1996. https://doi.org/10.1007/s10915-019-01011-3

\section{Published in:}

Journal of Scientific Computing

\section{Citing this paper}

Please note that where the full-text provided on Manchester Research Explorer is the Author Accepted Manuscript or Proof version this may differ from the final Published version. If citing, it is advised that you check and use the publisher's definitive version.

\section{General rights}

Copyright and moral rights for the publications made accessible in the Research Explorer are retained by the authors and/or other copyright owners and it is a condition of accessing publications that users recognise and abide by the legal requirements associated with these rights.

\section{Takedown policy}

If you believe that this document breaches copyright please refer to the University of Manchester's Takedown Procedures [http://man.ac.uk/04Y6Bo] or contact uml.scholarlycommunications@manchester.ac.uk providing relevant details, so we can investigate your claim.

\section{OPEN ACCESS}




\title{
Extension of tensor-product generalized and dense-norm summation-by-parts operators to curvilinear coordinates
}

\author{
David C. Del Rey Fernández • Pieter B. \\ Boom - Mark H. Carpenter - David W. \\ Zingg
}

Received: date / Accepted: date

\begin{abstract}
Methodologies are presented that enable the construction of provably linearly stable and conservative high-order discretizations of partial differential equations in curvilinear coordinates based on generalized summationby-parts operators, including operators with dense-norm matrices. Specifically, three approaches are presented for the construction of stable and conservative schemes in curvilinear coordinates using summation-by-parts (SBP) operators that have a diagonal norm but may or may not include boundary nodes: 1) the mortar-element approach, 2) the global SBP operator approach, and 3) the staggered grid approach. Moreover, the staggered grid approach is extended to enable the development of stable dense-norm operators in curvilinear coordinates. In addition, collocated upwind simultaneous approximation terms for the weak imposition of boundary conditions or inter-element coupling are extended to curvilinear coordinates with the new approaches. While the emphasis in the paper is on tensor-product SBP operators, the approaches that are covered are directly applicable to multidimensional SBP operators.
\end{abstract}

Portions of this paper were previously published in "Numerical Investigation of TensorProduct Summation-by-Parts Discretization Strategies and Operators" AIAA paper 20170530

D. C. Del Rey Fernández

NASA LaRC and NIA

E-mail: dcdelrey@gmail.com

P. D. Boom

UTIAS

E-mail: pieter.boom@mail.utoronto.ca

M. H. Carpenter

NASA LaRC

E-mail: mark.h.carpenter@nasa.gov

D. W. Zingg

UTIAS

E-mail:dwz@utias.utoronto.ca 
Keywords summation by parts - simultaneous approximation terms · curvilinear coordinates $\cdot$ linear stability

Mathematics Subject Classification (2010) MSC 65M06 · MSC 65M60 . MS C65M70

\section{Introduction}

The summation-by-parts (SBP) framework provides a systematic methodology for the development and analysis of stable and conservative methods for linear partial differential equations (PDEs) with variable coefficients (see the review papers $[20,46])$. SBP operators are matrix difference operators that are mimetic of integration by parts (IBP). The mimetic property of SBP operators is transferred to the complete discretization by combining SBP operators with appropriate procedures for coupling and imposition of boundary conditions (e.g. simultaneous approximation terms (SATs) $[5,6,36,37,7]$ ); in doing so, stability is proven in a one-to-one correspondence with continuous stability proofs. The SBP framework is attractive for the design and analysis of numerical methods because stability and conservation proofs reduce to the examination of matrices and their properties. This makes the approach applicable to the analysis of practical implementations of numerical algorithms, i.e., numerical integration is easily included in the analysis. Moreover, the framework is accessible to a broad range of researchers because it only requires simple matrix algebra, for the discrete analysis, and introductory level calculus, for the continuous analysis.

In recent years, there has been a sustained effort to extend the SBP concept to include a broader set of existing and novel discretization strategies, including continuous and discontinuous Galerkin $[31,17]$ and flux reconstruction $[42,41]$ methods as well as extensions to the analysis of nonlinear PDEs and general element types such as tetrahedra $[26,17,3,40,4,39,33,21,14,16,10$, 19]. The resulting generalizations allow for the construction of schemes having the SBP property on fairly arbitrary nodal distributions including those that do not have nodes on the boundaries (this implies that the surface mass matrix is not collocated). For curvilinear coordinates, until recently, it was unclear how to prove conservation for schemes constructed with SBP operators that do not have collocated surface mass matrices. However, this issue was solved by Crean et al. [16], in the context of multidimensional SBP operators (the solution of which was applied in the context of $p$-refinement on tensorproduct operators by Ref. [18]). Another outstanding issue is construction of stable schemes using SBP operators that have dense norms (mass matrix) in curvilinear coordinates. Svärd [45] proves that, in general, a valid norm cannot be constructed, and therefore energy stability cannot be proven, because the norm matrix does not commute with the diagonal metric Jacobian matrix. However, in the context of modal based schemes, the modal decoupled SBP operators of Chan $[10,11]$ result in provably stable discretizations for dense-norm 
operators. This approach circumvents the issues in Svärd [45] by incorporating the metric Jacobian into the norm matrix. The resulting schemes are the modal equivalent of the staggered approach presented here (see also the work of Chan et al. on the weight adjusted discontinuous Galerkin approach $[12,9$, 13]). Alternatively, Ranocha et al. [43] examine one-dimensional PDEs with a varying metric Jacobian and construct dense matrices, J, such that $J \frac{\mathrm{d} u}{\mathrm{~d} t}$ approximates the metric Jacobian times the time rate of change of the solution. In order to obtain stability, the matrix $\mathrm{J}$ is constructed such that when multiplied by the mass matrix, $\mathrm{H}, \mathrm{HJ}=\mathrm{J}^{\mathrm{T}} \mathrm{H}$ and $\mathrm{HJ}$ is symmetric-positive definite.

In this paper, the focus is on nodal SBP operators and construction of stable and conservative schemes in curvilinear coordinates. In particular, the objective is to present a number of alternative methods to handle diagonalnorm generalized SBP (GSBP) operators that do not have a collocated surface mass matrix and SBP operators with a dense norm. For diagonal-norm GSBP operators, several constructions that lead to stable and conservative schemes in curvilinear coordinates are presented, specifically:

- the mortar-element approach $[30,18]$ that require metric approximations based on Crean et al. [16]

- the global SBP approach

- the staggered grid approach $[39,19]$

In addition, the staggered grid approach is extended for the construction of stable and conservative approximations in curvilinear coordinates using densenorm SBP operators. While we emphasize tensor-product SBP operators, the developments in this paper can be directly applied to multidimensional SBP operators. Moreover, because the resulting schemes have the SBP property, they can be extended to entropy stable schemes for nonlinear conservation laws $[25,28,27,3,39,8,8,53,16,14,10,50,24,51,49,32,52]$.

The paper is organized as follows: the notation that is used is summarized in Section 2 and a brief review of SBP operators is given. To motivate the semi-discrete stability analysis, the continuous stability analysis of the convection equation in curvilinear coordinates is reviewed in Section 3. The schemes developed in this paper are provably element-wise conservative in curvilinear coordinates and this is discussed in Section 4. Several approaches for constructing stable and conservative discretizations using diagonal-norm SBP operators are reviewed in Section 5 and presented and analyzed in Sections 6 and 7. Approximation of the metric terms that arise from transforming PDEs into curvilinear coordinates such that a freestream is preserved is detailed in Section 8. The staggered-grid approach is reviewed in Section 9. This is followed by extension of the staggered-grid approach to dense-norm SBP operators (Section 10). Compatible SATs for interface and boundary conditions are presented in Section 11. A small set of numerical examples to verify the theory presented in the paper are given in Section 12. Finally, conclusions are drawn in Section 13. 


\begin{tabular}{ccc}
$(i, j, k)$ & $\min / \max$ & face number \\
\hline$i$ & $\min$ & 1 \\
$i$ & $\max$ & 2 \\
$j$ & $\min$ & 3 \\
$j$ & $\max$ & 4 \\
$k$ & $\min$ & 5 \\
$k$ & $\max$ & 6 \\
\hline
\end{tabular}

Table 1 Face numbering convention

\section{Notation, definitions, and review of SBP operators}

The notation used in this paper is consistent with that in Refs. [18,21,33]; readers familiar with that notation and SBP operators can skip to Section 3. Consider a hexahedral physical domain $\Omega \subset \mathbb{R}^{3}$, with boundary $\partial \Omega$, in Cartesian coordinates $\left(x_{1}, x_{2}, x_{3}\right) \subset \mathbb{R}^{3}$. The domain, $\Omega$, is partitioned into $K$ nonoverlapping hexahedral elements, $\Omega_{\kappa}, \kappa=1, \ldots, K$. Associating the index 3 -tuple $(i, j, k)$ with the computational coordinate 3 -tuple $\left(\xi_{1}, \xi_{2}, \xi_{3}\right)$, then the face numbering convention used in this paper is given in Table 2 .

The domain of the $\kappa^{\text {th }}$ element is denoted by $\Omega_{\kappa}$ and has boundary $\Gamma_{\kappa} \equiv$ $\partial \Omega_{\kappa}$. In order to use differentiation matrices constructed on a reference element, PDEs are solved in curvilinear coordinates, $\left(\xi_{1}, \xi_{2}, \xi_{3}\right) \subset \mathbb{R}^{3}$, where each $\Omega_{\kappa}$ is locally transformed to the computational domain $\hat{\Omega}_{\kappa}$ (e.g. the reference element) with boundary $\hat{\Gamma}_{\kappa} \equiv \partial \hat{\Omega}_{\kappa}$, under the following assumption:

Assumption 1 Each element in physical space is transformed using local and invertible curvilinear coordinate transformations that are compatible at shared interfaces, meaning that points in analogous locations in computational space on either side of the shared surface are mapped to the same physical location.

Matrices are presented using capital letters in sans-serif font, for example A, while vectors are denoted with lower-case bold font, for example $\boldsymbol{\xi}_{1}=$ $\left[\xi_{1,1}, \ldots, \xi_{1, N}\right]^{\mathrm{T}}$. Continuous functions on a space-time domain are represented by capital letters with script type. For example,

$$
\mathcal{U}\left(\xi_{1}, \xi_{2}, \xi_{3}, t\right) \in L^{2}\left(\left[\alpha_{\xi_{1}}, \beta_{\xi_{1}}\right] \times\left[\alpha_{\xi_{2}}, \beta_{\xi_{2}}\right] \times\left[\alpha_{\xi_{3}}, \beta_{\xi_{3}}\right] \times[0, T]\right)
$$

denotes a square integrable function. The restriction of such functions onto a grid is represented by lower-case bold font; for example the restriction of $\mathcal{U}$ onto a grid of $N_{1} \times N_{2} \times N_{3}$ nodes is given by the vector

$$
\boldsymbol{u}=\left[\mathcal{U}\left(\xi_{1,1}, \xi_{2,1}, \xi_{3,1}, t\right), \ldots, \mathcal{U}\left(\xi_{1, N_{1}}, \xi_{1, N_{2}}, \xi_{3, N_{3}}, t\right)\right]^{\mathrm{T}} .
$$

\subsection{Review of tensor-product SBP operators}

In this paper, one-dimensional SBP operators are extended to multiple dimensions using tensor products. 
1 Definition 1 The tensor product between matrices $\mathrm{A} \in \mathbb{R}^{n \times m}$ and $\mathrm{B} \in \mathbb{R}^{p \times q}$ is denoted by

$$
\mathrm{A} \otimes \mathrm{B} \equiv\left[\begin{array}{ccc}
a_{1,1} \mathrm{~B} \ldots & a_{1, m} \mathrm{~B} \\
\vdots & & \vdots \\
a_{n, 1} \mathrm{~B} & \ldots & a_{n, m} \mathrm{~B}
\end{array}\right]
$$

and has the following useful properties:

$-(A \otimes B)^{T}=A^{T} \otimes B^{T}$

$-(A \otimes B)^{-1}=A^{-1} \otimes B^{-1}$,

- if the products $A C$ and $B D$ exist then $(A \otimes B)(C \otimes D)=A C \otimes B D$.

SBP operators are matrix difference operators approximating derivatives at mesh nodes; they can be characterized in terms of their degree, which is the degree of monomial that they differentiate exactly. In this paper, the word order is reserved to refer to the order of the solution error and the accuracy of the discretization is exclusively discussed in terms of degree. The definition of a one-dimensional SBP operator in the $\xi_{l}$ direction is $[17,20,46]$ :

Definition 2 Summation-by-parts operator for the first derivative: A matrix operator, $\mathrm{D}_{\xi_{l}}^{(1 D)} \in \mathbb{R}^{N_{l} \times N_{l}}$, is an SBP operator approximating the derivative $\frac{\partial}{\partial \xi_{l}}$ on the nodal distribution $\xi_{l} \in\left[\alpha_{\xi_{l}}, \beta_{\xi_{l}}\right]$, of degree $\mathrm{p}$ if

1. $\mathrm{D}_{\xi_{l}}^{(1 D)} \boldsymbol{\xi}_{l}^{k}=k \boldsymbol{\xi}_{l}^{k-1}, k=0,1, \ldots, p ;$

2. $\mathrm{D}_{\xi_{l}}^{(1 D)} \equiv\left(\mathrm{H}_{\xi_{l}}^{(1 D)}\right)^{-1} \mathrm{Q}_{\xi_{l}}^{(1 D)}$, where the norm matrix, $\mathrm{H}_{\xi_{l}}^{(1 D)}$, is symmetric positive definite, and;

3. $\mathrm{Q}_{\xi_{l}}^{(1 D)}=\left(\mathrm{S}_{\xi_{l}}^{(1 D)}+\frac{1}{2} \mathrm{E}_{\xi_{l}}^{(1 D)}\right)$, where $\mathrm{S}_{\xi_{l}}^{(1 D)}=-\left(\mathrm{S}_{\xi_{l}}^{(1 D)}\right)^{\mathrm{T}}, \mathrm{E}_{\xi_{l}}^{(1 D)}=\left(\mathrm{E}_{\xi_{l}}^{(1 D)}\right)^{\mathrm{T}}$, and $\mathrm{E}_{\xi_{l}}^{(1 D)}$ satisfies

$$
\left(\boldsymbol{\xi}_{l}^{i}\right)^{\mathrm{T}} \mathrm{E}_{\xi_{l}}^{(1 D)} \boldsymbol{\xi}_{l}^{j}=\beta_{\xi_{l}}^{i+j}-\alpha_{\xi_{l}}^{i+j}, \quad i, j=0,1, \ldots, r, \quad r \geq p .
$$

For the purpose of imposing boundary conditions or inter-element coupling using SATs, it is convenient to decompose $\mathrm{E}_{\xi_{l}}^{(1 D)}$ as [17]

$$
\mathrm{E}_{\xi_{l}}^{(1 D)}=\boldsymbol{t}_{\beta_{\xi_{l}}} \boldsymbol{t}_{\beta_{\xi_{l}}}^{\mathrm{T}}-\boldsymbol{t}_{\alpha_{\xi_{l}}} \boldsymbol{t}_{\alpha_{\xi_{l}}}^{\mathrm{T}}
$$

where

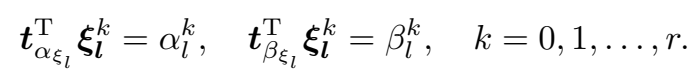

The tensor-product extension to three dimensions of the first-derivative SBP operators is given as

$$
\mathrm{D}_{\xi_{1}} \equiv \mathrm{D}_{\xi_{1}}^{(1 D)} \otimes \mathrm{I}_{\xi_{2}} \otimes \mathrm{I}_{\xi_{3}}, \quad \mathrm{D}_{\xi_{2}} \equiv \mathrm{I}_{\xi_{1}} \otimes \mathrm{D}_{\xi_{2}}^{(1 D)} \otimes \mathrm{I}_{\xi_{3}}, \quad \mathrm{D}_{\xi_{2}} \equiv \mathrm{I}_{\xi_{1}} \otimes \mathrm{I}_{\xi_{2}} \otimes \mathrm{D}_{\xi_{3}}^{(1 D)}
$$

where $\mathrm{I}_{\xi_{l}} \in \mathbb{R}^{N_{l} \times N_{l}}$ are identity matrices for $l=1,2,3$. 
For the purpose of analysis, it is convenient to rewrite the tensor-product operators as multidimensional SBP operators; for example

$$
\begin{aligned}
& \mathrm{D}_{\xi_{1}} \equiv \mathrm{H}^{-1} \mathrm{Q}_{\xi_{1}}, \quad \mathrm{Q}_{\xi_{1}} \equiv \mathrm{Q}_{\xi_{1}}^{(1 D)} \otimes \mathrm{H}_{\xi_{2}}^{(1 D)} \otimes \mathrm{H}_{\xi_{3}}^{(1 D)}, \\
& \mathrm{H} \equiv \mathrm{H}_{\xi_{1}}^{(1 D)} \otimes \mathrm{H}_{\xi_{2}}^{(1 D)} \otimes \mathrm{H}_{\xi_{3}}^{(1 D)} \\
& \mathrm{E}_{\xi_{1}} \equiv \mathrm{E}_{\beta_{\xi_{1}}}+\mathrm{E}_{\alpha_{\xi_{1}}}, \quad \mathrm{E}_{\beta_{\xi_{1}}} \equiv \mathrm{R}_{\beta_{\xi_{1}}}^{\mathrm{T}} \mathrm{H}_{\xi_{1}}^{\perp} \mathrm{R}_{\beta_{\xi_{1}}}, \quad \mathrm{E}_{\alpha_{\xi_{1}}} \equiv-\mathrm{R}_{\alpha_{\xi_{1}}}^{\mathrm{T}} \mathrm{H}_{\xi_{1}}^{\perp} \mathrm{R}_{\alpha_{\xi_{1}}}, \\
& \mathrm{H}_{\xi_{1}}^{\perp} \equiv \mathrm{H}_{\xi_{2}}^{(1 D)} \otimes \mathrm{H}_{\xi_{3}}^{(1 D)}, \\
& \mathrm{R}_{\beta_{\xi_{1}}} \equiv \boldsymbol{t}_{\beta_{\xi_{1}}}^{\mathrm{T}} \otimes \mathrm{I}_{\xi_{2}} \otimes \mathrm{I}_{\xi_{3}}, \quad \mathrm{R}_{\alpha_{\xi_{1}}} \equiv \boldsymbol{t}_{\alpha_{\xi_{1}}}^{\mathrm{T}} \otimes \mathrm{I}_{\xi_{2}} \otimes \mathrm{I}_{\xi_{3}} .
\end{aligned}
$$

To clarify the meaning of each of the constituent matrices, i.e., $\mathrm{H}, \mathrm{Q}_{\xi_{1}}, \mathrm{E}_{\xi_{1}}$, etc., we link them here to various bilinear forms. Consider the vectors $\boldsymbol{u}$ and $\boldsymbol{v}$ constructed from the evaluation of two continuous functions $\mathcal{U}$ and $\mathcal{V}$ on the nodes of a tensor-product hexahedral element in computational coordinates, then

$$
\begin{aligned}
& \boldsymbol{v}_{\kappa}^{\mathrm{T}} \mathrm{H} \boldsymbol{u}_{\kappa} \approx \int_{\hat{\Omega}_{\kappa}} \mathcal{V}_{\kappa} \mathcal{U}_{\kappa} \mathrm{d} \hat{\Omega}, \quad \boldsymbol{v}_{\kappa}^{\mathrm{T}} \mathrm{Q}_{\xi_{1}} \boldsymbol{u}_{\kappa} \approx \int_{\hat{\Omega}_{\kappa}} \mathcal{V}_{\kappa} \frac{\partial \mathcal{U}_{\kappa}}{\partial \xi_{1}} \mathrm{~d} \hat{\Omega}, \quad \boldsymbol{v}_{\kappa}^{\mathrm{T}} \mathrm{E}_{\xi_{1}} \boldsymbol{u}_{\kappa} \approx \oint_{\hat{\Gamma}_{\kappa}} \mathcal{V}_{\kappa} \mathcal{U}_{\kappa} n_{\xi_{1}} \mathrm{~d} \hat{\Gamma}, \\
& \boldsymbol{v}_{\kappa}^{\mathrm{T}} \mathrm{E}_{\alpha_{\xi_{1}}} \boldsymbol{u}_{\kappa} \approx \oint_{\hat{\Gamma}_{\kappa}^{\alpha} \xi_{1}} \mathcal{V}_{\kappa} \mathcal{U}_{\kappa} n_{\xi_{1}} \mathrm{~d} \hat{\Gamma}, \quad \boldsymbol{v}_{\kappa}^{\mathrm{T}} \mathrm{E}_{\beta_{\xi_{1}}} \boldsymbol{u}_{\kappa} \approx \oint_{\hat{\Gamma}_{\kappa}^{\beta} \xi_{1}} \mathcal{V}_{\kappa} \mathcal{U}_{\kappa} n_{\xi_{1}} \mathrm{~d} \hat{\Gamma}
\end{aligned}
$$

where $n_{\xi_{1}}$ is the $\xi_{1}$ component of the unit normal, and $\hat{\Gamma}_{\kappa}^{\alpha \xi_{1}}$ and $\hat{\Gamma}_{\kappa}^{\beta_{\xi_{1}}}$ are the surfaces perpendicular to the $\xi_{1}$ coordinate. Furthermore, the operators $\mathrm{R}_{\alpha_{\xi_{1}}}$ and $\mathrm{R}_{\beta_{\xi_{1}}}$ interpolate/extrapolate to opposing surfaces, i.e.,

$$
\mathrm{R}_{\alpha_{\xi_{1}}} \boldsymbol{u}_{\kappa} \approx \mathcal{U}_{\kappa}\left(\boldsymbol{\xi}_{1}^{\alpha_{\xi_{1}}}, \boldsymbol{\xi}_{2}^{\alpha_{\xi_{1}}}, \boldsymbol{\xi}_{3}^{\alpha_{\xi_{1}}}\right) \quad \mathrm{R}_{\beta_{\xi_{1}}} \boldsymbol{u}_{\kappa} \approx \mathcal{U}_{\kappa}\left(\boldsymbol{\xi}_{1}^{\beta_{\xi_{1}}}, \boldsymbol{\xi}_{2}^{\beta_{\xi_{1}}}, \boldsymbol{\xi}_{3}^{\beta_{\xi_{1}}}\right)
$$

where $\boldsymbol{\xi}_{i}^{\alpha_{\xi_{1}}}$ and $\boldsymbol{\xi}_{i}^{\beta_{\xi_{1}}}, i=1,2,3$, are the coordinates of the nodes at the $\hat{\Gamma}_{\kappa}^{\alpha_{\xi_{1}}}$ and $\hat{\Gamma}_{\kappa}^{\beta_{\xi_{1}}}$ surface respectively.

\section{Linear stability analysis of the convection equation in curvilinear coordinates}

The combination of SBP-SATs to discretize PDEs in a stable fashion is based on the idea of mimicking, in a one-to-one fashion, the linear and nonlinear stability proofs available for continuous PDEs. To motivate the semi-discrete 
stability analysis that appears later in this paper, the stability analysis of the three-dimensional periodic convection equation,

$$
\frac{\partial \mathcal{U}}{\partial t}+\sum_{m=1}^{3} \frac{\partial \mathcal{U}}{\partial x_{m}}=0, \quad \forall\left(x_{1}, x_{2}, x_{3}\right) \in[0,1]^{3}=\Omega, \quad t \geq 0
$$

is reviewed.

The initial and boundary conditions are

$$
\begin{aligned}
& \mathcal{U}\left(x_{1}, x_{2}, x_{3}, 0\right)=\mathcal{U}_{0}\left(x_{1}, x_{2}, x_{3}\right), \\
& \mathcal{U}\left(0, x_{2}, x_{3}, t\right)=\mathcal{U}\left(1, x_{2}, x_{3}, t\right) \\
& \mathcal{U}\left(x_{1}, 0, x_{3}, t\right)=\mathcal{U}\left(x_{1}, 1, x_{3}, t\right), \quad \forall\left(x_{1}, x_{2}, x_{3}\right) \in[0,1]^{3}, \quad t \geq 0, \\
& \mathcal{U}\left(x_{1}, x_{2}, 0, t\right)=\mathcal{U}\left(x_{1}, x_{2}, 1, t\right) .
\end{aligned}
$$

The domain $\Omega$ is partitioned into $K$ sub-domains $\Omega_{\kappa}$ and on each a timeinvariant curvilinear coordinate transformation is applied to (2). Thus, in strong conservation form, on the $\kappa^{\text {th }}$ element (2) becomes

$$
\mathcal{J}_{\kappa} \frac{\partial \mathcal{U}_{\kappa}}{\partial t}+\sum_{l, m=1}^{3} \frac{\partial}{\partial \xi_{l}}\left(\mathcal{J}_{\kappa} \frac{\partial \xi_{l}}{\partial x_{m}} \mathcal{U}_{\kappa}\right)=0, \quad\left(\xi_{1}, \xi_{2}, \xi_{3}\right) \in \hat{\Omega}_{\kappa}
$$

where the metric invariants have been used to obtain this form:

$$
\sum_{l=1}^{3} \frac{\partial}{\partial \xi_{l}}\left(\mathcal{J}_{\kappa} \frac{\partial \xi_{l}}{\partial x_{m}}\right)=0, \quad m=1,2,3,
$$

and $\mathcal{J}_{\kappa} \equiv \operatorname{det}\left(\frac{\partial\left(\mathrm{x}_{1}, \mathrm{x}_{2}, \mathrm{x}_{3}\right)^{\mathrm{T}}}{\partial\left(\xi_{1}, \xi_{2}, \xi_{3}\right)^{\mathrm{T}}}\right)$. By noting that

$$
\begin{aligned}
& \sum_{\substack{l=1 \\
m=1,2,3}}^{3} \frac{\partial}{\partial \xi_{l}}\left(\mathcal{J}_{\kappa} \frac{\partial \xi_{l}}{\partial x_{m}} \mathcal{U}_{\kappa}\right)=\sum_{l=1}^{3} \frac{1}{2} \frac{\partial}{\partial \xi_{l}}\left(\mathcal{J}_{\kappa} \frac{\partial \xi_{l}}{\partial x_{m}} \mathcal{U}_{\kappa}+\frac{1}{2} \mathcal{J}_{\kappa} \frac{\partial \xi_{l}}{\partial x_{m}} \frac{\partial \mathcal{U}_{\kappa}}{\partial \xi_{l}}\right), \\
& m=1
\end{aligned}
$$

where the metric invariants (5) have again been used, the conservative form of the convection equation (4) is recast in skew-symmetric form [28]

$$
\mathcal{J}_{\kappa} \frac{\partial \mathcal{U}_{\kappa}}{\partial t}+\frac{1}{2} \sum_{l, m=1}^{3}\left\{\frac{\partial}{\partial \xi_{l}}\left(\mathcal{J}_{\kappa} \frac{\partial \xi_{l}}{\partial x_{m}} \mathcal{U}_{\kappa}\right)+\mathcal{J}_{\kappa} \frac{\partial \xi_{l}}{\partial x_{m}} \frac{\partial \mathcal{U}_{\kappa}}{\partial \xi_{l}}\right\}=0
$$

This form is important in constructing a provably stable semi-discrete form. Furthermore, it will be shown that in order to preserve a freestream and element-wise conservation, the discrete metrics must satisfy the metric invariant condition discretely. 
Now, the energy method is used to show that the problem defined by (6) (or equivalently (4)) with data (3) is stable. Multiplying (6) by the solution and integrating in space results in

$$
\begin{aligned}
\int_{\hat{\Omega}_{\kappa}}\left(\mathcal{U}_{\kappa} \mathcal{J}_{\kappa} \frac{\partial \mathcal{U}_{\kappa}}{\partial t}\right) \mathrm{d} \hat{\Omega}+ \\
\quad \int_{\hat{\Omega}_{\kappa}}\left(\frac{\mathcal{U}_{\kappa}}{2} \sum_{l, m=1}^{3}\left\{\frac{\partial}{\partial \xi_{l}}\left(\mathcal{J}_{\kappa} \frac{\partial \xi_{l}}{\partial x_{m}} \mathcal{U}_{\kappa}\right)+\mathcal{J}_{\kappa} \frac{\partial \xi_{l}}{\partial x_{m}} \frac{\partial \mathcal{U}_{\kappa}}{\partial \xi_{l}}\right\}\right) \mathrm{d} \hat{\Omega}=0
\end{aligned}
$$

For the temporal term, the equality $\mathcal{U}_{\kappa} \mathcal{J}_{\kappa} \frac{\partial \mathcal{U}_{\kappa}}{\partial t}=\frac{1}{2} \frac{\partial \mathcal{J}_{\kappa}\left(\mathcal{U}_{\kappa}\right)^{2}}{\partial t}$ and then the Leibniz rule are used to bring the derivative outside of the integral. For the spatial term, integration by parts is used, which results in

$$
\frac{\mathrm{d}}{\mathrm{d} t}\left\|\mathcal{U}_{\kappa}\right\|_{\mathcal{J}_{\kappa}}^{2}+\oint_{\hat{\Gamma}_{\kappa}}\left\{\sum_{l, m=1}^{3} \mathcal{J}_{\kappa} \frac{\partial \xi_{l}}{\partial x_{m}}\left(\mathcal{U}_{\kappa}\right)^{2} n_{\xi_{l}}\right\} \mathrm{d} \hat{\Gamma}=0
$$

where $n_{\xi_{l}}$ is the $\xi_{l}$ component of the outward pointing unit normal and the following norm is introduced:

$$
\left\|\mathcal{U}_{\kappa}\right\|_{\mathcal{J}_{\kappa}}^{2} \equiv \int_{\hat{\Omega}_{\kappa}} \mathcal{U}_{\kappa}^{2} \mathcal{J}_{\kappa} \mathrm{d} \hat{\Omega}
$$

Applying the boundary conditions, integrating in time, applying the initial condition, and rearranging gives

$$
\left\|\mathcal{U}_{\kappa}\right\|_{\mathcal{J}_{\kappa}}^{2}=\left\|\mathcal{U}_{0}\right\|_{\mathcal{J}_{\kappa}}^{2}
$$

which shows that the solution is bounded by the data and therefore the problem is stable.

\section{Conservation}

The discussion in this section follows closely that in Ref. [18]. Our interest is in solving nonlinear conservation laws; this implies that in finite time non-smooth solutions can result even when the conservation law is closed with smooth data. In the case of the linear variable coefficient hyperbolic conservation laws that are analyzed in this paper, non-smooth solutions can only occur as a result of the initial condition or the boundary conditions. In order to accommodate non-smooth solutions it is necessary to consider the weak form of the conservation law. The importance of the weak form is that it supports a restricted class of discontinuous solutions, i.e., those that support the Rankine-Hugoniot conditions. 
In this paper, semi-discrete forms are analyzed; thus, the appropriate weak form is

$$
\begin{aligned}
\int_{\hat{\Omega}_{\kappa}} & \left(\mathcal{V}_{\kappa} \frac{\partial \mathcal{U}_{\kappa}}{\partial t} \mathcal{J}_{\kappa}-\sum_{l, m=1}^{3} \mathcal{J}_{\kappa} \frac{\partial \xi_{l}}{\partial x_{m}} \mathcal{F}_{x_{m}} \frac{\partial \mathcal{V}_{\kappa}}{\partial \xi_{l}}\right) \mathrm{d} \hat{\Omega}+ \\
& \oint_{\hat{\Gamma}_{\kappa}} \mathcal{V}_{\kappa} \sum_{l, m=1}^{3} \mathcal{J}_{\kappa} \frac{\partial \xi_{l}}{\partial x_{m}} \mathcal{F}_{x_{m}} n_{\xi_{l}} \mathrm{~d} \hat{\Gamma}=\mathbf{0}, \quad t \geq 0, \quad \kappa=1,2, \ldots, K,
\end{aligned}
$$

for all test functions $\mathcal{V}_{\kappa} \in \mathrm{C}^{1}\left(\hat{\Omega}_{\kappa}\right)$. The flux function $\mathcal{F}_{x_{m}}$ in the current context is a scalar and is equal to $\mathcal{U}_{\kappa}$.

At its core, the analysis that is employed relies on the Lax-Wendroff Theorem [35]. To use this theorem, it is necessary to recast the schemes in telescoping flux form, which in one dimension at node $j$ over a control volume $\left[x_{j-\frac{1}{2}}, x_{j+\frac{1}{2}}\right]$ reads

$$
\frac{\mathrm{d} u_{j}}{\mathrm{~d} t}+\frac{\left(g_{j+\frac{1}{2}}-g_{j-\frac{1}{2}}\right)}{\Delta x}=0,
$$

where $g$ is a unique general scalar flux function at the boundaries of the control volume and $\Delta x$ is the mesh spacing. If a scheme can be recast in this way it is said to be conservative.

\subsection{Element-wise conservation}

Here, the necessary tools are laid out to prove that the schemes have a telescoping flux form at the element level. Recently, Shi and Shu [44] presented an extension of the Lax-Wendroff Theorem [35] applicable to element-wise conservation analysis for general multidimensional discretizations. Here, use is made of Shi and Shu's framework, as applied to curvilinear coordinates and SBP operators as given in Ref. [18]. It is necessary to show that the semi-discrete equations satisfy the following:

- Telescoping form: The schemes can be algebraically manipulated into a general telescoping flux form at the element level given by

$$
\frac{\mathrm{d} \bar{u}_{\kappa}}{\mathrm{d} t}+\sum_{l=1}^{3}\left(g_{\kappa}^{(2 l-1)}+g_{\kappa}^{(2 l)}\right)=0
$$

where $\bar{u}_{\kappa}$ is a generalized locally conserved quantity and $g_{\kappa}^{(2 l-1)}$ and $g_{\kappa}^{(2 l)}$ are generalized fluxes on the $(2 l-1)$ and $2 l$ faces of the element, respectively. A necessary condition for the scheme to have the telescoping property is that fluxes on any interface are unique. 
- Consistency: For a constant flow $\mathcal{U}=\mathcal{U}_{c}$

$$
\begin{aligned}
& \bar{u}_{\kappa}=\left(\int_{\hat{\Omega}_{\kappa}} \mathcal{J}_{\kappa} \mathrm{d} \hat{\Omega}+\mathcal{O}(h)\right) \mathcal{U}_{c} \\
& g_{\kappa}^{(2 l-1)}=\sum_{m=1}^{3}\left(\oint_{\hat{\Gamma}_{\kappa}}^{(2 l-1)} \mathcal{J}_{\kappa} \frac{\partial \xi_{l}}{\partial x_{m}} n_{\xi_{l}} \mathrm{~d} \hat{\Gamma}+\mathcal{O}(h)\right) \mathcal{F}_{x_{m}}\left(\mathcal{U}_{c}\right), \\
& g_{\kappa}^{(2 l)}=\sum_{m=1}^{3}\left(\oint_{\hat{\Gamma}_{\kappa}}^{(2 l)} \mathcal{J}_{\kappa} \frac{\partial \xi_{l}}{\partial x_{m}} n_{\xi_{l}} \mathrm{~d} \hat{\Gamma}+\mathcal{O}(h)\right) \mathcal{F}_{x_{m}}\left(\mathcal{U}_{c}\right),
\end{aligned}
$$

where $h$ is an appropriate measure of the element size.

- Boundedness: The generalized conserved quantity and fluxes are bounded in terms of the $L^{\infty}$ norm of the numerical solution:

$$
\begin{aligned}
& \left|\bar{u}_{\kappa}\left(\boldsymbol{u}_{h}\right)-\bar{v}_{\kappa}\left(\boldsymbol{v}_{h}\right)\right| \leq C h^{d}\left\|\boldsymbol{u}_{h}-\boldsymbol{v}_{h}\right\|_{L^{\infty}\left(B_{\kappa}\right)}, \\
& \left|g_{\kappa}^{(2 l-1)}\left(\boldsymbol{u}_{h}\right)-g_{\kappa}^{(2 l-1)}\left(\boldsymbol{v}_{h}\right)\right| \leq C h^{d-1}\left\|\boldsymbol{u}_{h}-\boldsymbol{v}_{h}\right\|_{L^{\infty}\left(B_{\kappa}\right)}, \\
& \left|g_{\kappa}^{(2 l)}\left(\boldsymbol{u}_{h}\right)-g_{\kappa}^{(2 l)}\left(\boldsymbol{v}_{h}\right)\right| \leq C h^{d-1}\left\|\boldsymbol{u}_{h}-\boldsymbol{v}_{h}\right\|_{L^{\infty}\left(B_{\kappa}\right)},
\end{aligned}
$$

where $\boldsymbol{u}_{h}$ and $\boldsymbol{v}_{h}$ are two discrete functions in the numerical solution space and $C$ is some positive constant. Moreover, $B_{\kappa} \equiv\left\{\boldsymbol{x} \in \mathbb{R}^{d}:\left|\boldsymbol{x}-\boldsymbol{x}_{c}\right|<c h\right\}$, $\boldsymbol{x}_{c}$ is the element center, c $(>1)$ is independent of mesh size, and $d$ is the spatial dimension (here $d=3$ ).

- Global conservation:

$$
\sum_{\kappa=1}^{K} \bar{u}_{\kappa}=\int_{\Omega} \mathcal{U} \mathrm{d} \Omega+\mathcal{O}(h) .
$$

Remark 1 The Shi and Shu framework is meant to deal with a very large class of schemes; however, in the current context, the generalized quantities approximate the following:

$$
\begin{aligned}
& \bar{u}_{\kappa} \approx \int_{\hat{\Omega}_{\kappa}} \mathcal{U} \mathcal{J}_{\kappa} \mathrm{d} \hat{\Omega}, \\
& g_{(2 l-1)} \approx \sum_{m=1}^{3} \oint_{\hat{\Gamma}_{\kappa}^{(2 l-1)}} \mathcal{J}_{\kappa} \frac{\partial \xi_{l}}{\partial x_{m}} \mathcal{F}_{x_{m}} n_{\xi_{l}} \mathrm{~d} \hat{\Gamma}, \\
& g_{2 l} \approx \sum_{m=1}^{3} \oint_{\hat{\Gamma}_{\kappa}^{(2 l)}} \mathcal{J}_{\kappa} \frac{\partial \xi_{l}}{\partial x_{m}} \mathcal{F}_{x_{m}} n_{\xi_{l}} \mathrm{~d} \hat{\Gamma} .
\end{aligned}
$$

\section{Overview of the discretization approaches}

The SBP framework provides a simple algebraic means of developing and analyzing numerical methods and in recent years has been extended along the lines of finite-element schemes. In that context, it is natural to introduce three 


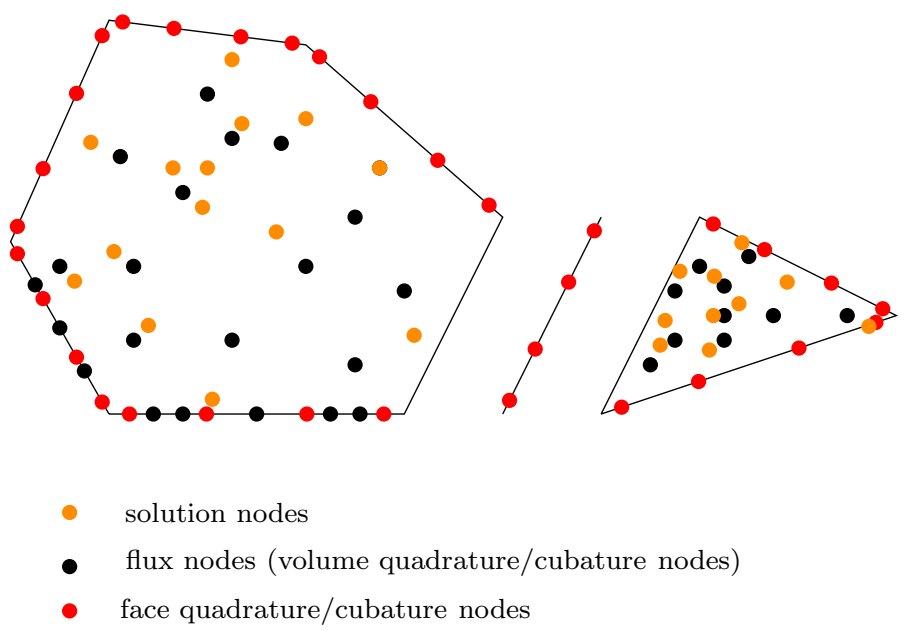

Fig. 1 The various nodes used to construct an SBP operator.

sets of nodes, a set of solution nodes where the unknowns exist, a set of volume quadrature/cubature nodes, where volume integrals are approximated (we will think of them as flux nodes where derivatives are approximated), and a set of surface quadrature/cubature nodes, where surface integrals are approximated (on these surfaces, inter-element coupling or boundary conditions are weakly imposed) see Figure 1. By combining different sets of nodes, different SBP schemes emerge; construction of energy stable approximations in curvilinear coordinates is the main focus of what follows. The extensions contained herein allow for the SBP framework to be applied to the design and analysis of a large set of existing and novel schemes with provable properties.

The first set of SBP operators that are consider are those where the solution nodes and volume quadrature nodes are collocated (by collocated we mean that these two sets of nodes are identical) but where the surface quadrature nodes are not. Two approaches are developed for constructing energy-stable discretizations in curvilinear coordinates. In the first, the surface quadrature nodes are conceptually located on a mortar element that resides between elements (the mortar element is one dimension lower than the volume elements, i.e. $1 \mathrm{D}=$ point, $2 \mathrm{D}=$ surface, and $3 \mathrm{D}=$ plane). On this mortar element, inter-element coupling and boundary conditions are weakly imposed. The main difficulty resides in the construction of the metric terms that result from the curvilinear coordinate transformation such that the resulting scheme is energy stable, conservative, and freestream preserving. Alternatively, for this same class of SBP operators, we consider constructing a global SBP operator over the entire mesh, which allows for well-known freestream-preserving procedures for the approximation of metric terms to be applied, such as the approach of Thomas and Lombard [47].

Next we consider using distinct solution and volume quadrature nodes but retain a diagonal-norm matrix (i.e., a mass lumped mass matrix which results 
1 by approximating the mass matrix using a quadrature rule on the solution nodes). The technical challenge with this approach is constructing interpolation/extrapolation operators from the solution nodes to the volume quadrature nodes and back so that the SBP property is preserved. The resulting schemes allow for the introduction of the concept of over-integration into the SBP framework.

Finally, again considering distinct solution and volume quadrature nodes, we consider the construction of stable SBP schemes where we do not mass lump, i.e. the mass matrix is no longer diagonal and again, the difficulty resides in how to construct proper interpolation/extrapolation operators.

\section{The mortar-element approach}

In this section, the construction of stable and conservative collocated GSBP discretizations of linear PDEs in curvilinear coordinates is discussed. The resulting discretization is an application of the work of Crean et al. [16] on multidimensional SBP schemes to tensor-product SBP schemes. The critical difficulty resides in how to construct the SATs and how to approximate the metric terms (this is accomplished by introducing a fictitious surface between abutting elements, the mortar element, on which the coupling terms are constructed). The discretization of (6) is given as

$$
\begin{aligned}
& \operatorname{diag}(\mathcal{J})_{\kappa} \frac{\mathrm{d} \boldsymbol{u}_{\kappa}}{\mathrm{d} t}+\frac{1}{2} \sum_{l, m=1}^{3}\left(\mathrm{D}_{\xi_{l}} \operatorname{diag}\left(\mathcal{J} \frac{\partial \xi_{l}}{\partial x_{m}}\right)_{\kappa}+\operatorname{diag}\left(\mathcal{J} \frac{\partial \xi_{l}}{\partial x_{m}}\right)_{\kappa} \mathrm{D}_{\xi_{l}}\right) \boldsymbol{u}_{\kappa}= \\
& \mathrm{H}^{-1} \sum_{l, m=1}^{3}\left\{\frac{1}{2} \mathrm{E}_{\xi_{l}} \operatorname{diag}\left(\mathcal{J} \frac{\partial \xi_{l}}{\partial x_{m}}\right)_{\kappa} \boldsymbol{u}_{\kappa}-\frac{1}{2}\left(\mathrm{E}_{2 l \mathrm{to}(2 l-1)}^{\kappa, m} \boldsymbol{u}_{(2 l-1)}+\mathrm{E}_{(2 l-1) \text { to } 2 l}^{\kappa, m} \boldsymbol{u}_{2 l}\right)\right\} .
\end{aligned}
$$

where, $\operatorname{diag}(\mathcal{J})_{\kappa}$ and $\operatorname{diag}\left(\mathcal{J} \frac{\partial \xi_{l}}{\partial x_{m}}\right)_{\kappa}$ are diagonal matrices with approximations to the metric Jacobian and metric terms along their diagonal, respectively (how these terms are approximated is dealt with later). Furthermore, $\boldsymbol{u}_{2 l}$ and $\boldsymbol{u}_{(2 l-1)}$ are the numerical solution in elements abutting the $2 l$ and $2 l-1$ interfaces, respectively. Moreover, the RHS of (15) is composed of the SATs used to weakly impose inter-element coupling or boundary conditions. The coupling terms of the SAT, for example, $\mathrm{E}_{(2 l-1) \text { to } 2 l}^{\kappa, m}$ which is associated with the metric terms $\mathcal{J}_{\kappa} \frac{\partial \xi_{l}}{\partial x_{m}}$, couples the $(2 l-1)$ face of the abutting element to the $2 l$ face of the $\kappa$ element. Moreover, these coupling terms are then constructed to a) preserve design order and b) so that skew-symmetry is maintained globally (the construction of the coupling terms so that skew-symmetry is preserved is discussed later).

To give further insight into the terms in (15), we recast into integral form by multiplying by the restriction of a test function, $\mathcal{V}_{\kappa}$, onto the element's solution 
nodes, $\boldsymbol{v}_{\kappa}$ and discretely integrating. This is accomplished by multiplying (15) by $\boldsymbol{v}_{\kappa}^{\mathrm{T}} \mathrm{H}$, resulting in

$\boldsymbol{v}_{\kappa}^{\mathrm{T}} \mathrm{H} \operatorname{diag}(\mathcal{J})_{\kappa} \frac{\mathrm{d} \boldsymbol{u}_{\kappa}}{\mathrm{d} t}$

$+\frac{1}{2} \boldsymbol{v}_{\kappa}^{\mathrm{T}} \mathrm{H} \sum_{l, m=1}^{3}\left(\mathrm{D}_{\xi_{l}} \operatorname{diag}\left(\mathcal{J} \frac{\partial \xi_{l}}{\partial x_{m}}\right)_{\kappa}+\operatorname{diag}\left(\mathcal{J} \frac{\partial \xi_{l}}{\partial x_{m}}\right)_{\kappa} \mathrm{D}_{\xi_{l}}\right) \boldsymbol{u}_{\kappa}=$

$\boldsymbol{v}_{\kappa}^{\mathrm{T}} \sum_{l, m=1}^{3}\left\{\frac{1}{2} \mathrm{E}_{\xi_{l}} \operatorname{diag}\left(\mathcal{J} \frac{\partial \xi_{l}}{\partial x_{m}}\right)_{\kappa} \boldsymbol{u}_{\kappa}-\frac{1}{2}\left(\mathrm{E}_{2 l \mathrm{to}(2 l-1)}^{\kappa, m} \boldsymbol{u}_{(2 l-1)}+\mathrm{E}_{(2 l-1) \mathrm{to} 2 l}^{\kappa, m} \boldsymbol{u}_{2 l}\right)\right\}$.

Each of the terms approximates the following bilinear forms:

$$
\begin{gathered}
\boldsymbol{v}_{\kappa}^{\mathrm{T}} \mathrm{H} \operatorname{diag}(\mathcal{J})_{\kappa} \frac{\mathrm{d} \boldsymbol{u}_{\kappa}}{\mathrm{d} t} \approx \int_{\hat{\Omega}_{\kappa}} \mathcal{V}_{\kappa} \mathcal{U}_{\kappa} \mathcal{J}_{\kappa} \mathrm{d} \hat{\Omega}, \\
\boldsymbol{v}_{\kappa}^{\mathrm{T}} \mathrm{H}\left(\mathrm{D}_{\xi_{l}} \operatorname{diag}\left(\mathcal{J} \frac{\partial \xi_{l}}{\partial x_{m}}\right)_{\kappa}+\operatorname{diag}\left(\mathcal{J} \frac{\partial \xi_{l}}{\partial x_{m}}\right)_{\kappa} \mathrm{D}_{\xi_{l}}\right) \boldsymbol{u}_{\kappa}= \\
\boldsymbol{v}_{\kappa}^{\mathrm{T}}\left(\mathrm{Q}_{\xi_{l}} \operatorname{diag}\left(\mathcal{J} \frac{\partial \xi_{l}}{\partial x_{m}}\right)_{\kappa}+\operatorname{diag}\left(\mathcal{J} \frac{\partial \xi_{l}}{\partial x_{m}}\right)_{\kappa} \mathrm{Q}_{\xi_{l}}\right) \boldsymbol{u}_{\kappa} \approx \\
\int_{\hat{\Omega}_{\kappa}}\left\{\mathcal{V}_{\kappa} \frac{\partial}{\partial \xi_{l}}\left(\mathcal{J}_{\kappa} \frac{\partial \xi_{l}}{\partial x_{m}} \mathcal{U}_{\kappa}\right)+\mathcal{V}_{\kappa} \mathcal{J}_{\kappa} \frac{\partial \xi_{l}}{\partial x_{m}} \frac{\partial \mathcal{U}_{\kappa}}{\partial \xi_{l}}\right\} \mathrm{d} \hat{\Omega}, \\
\frac{1}{2} \boldsymbol{v}_{\kappa}^{\mathrm{T}} \mathrm{E}_{\xi_{l}} \operatorname{diag}\left(\mathcal{J} \frac{\partial \xi_{l}}{\partial x_{m}}\right)_{\kappa} \boldsymbol{u}_{\kappa} \approx \oint_{\hat{\Gamma}_{\kappa}} \mathcal{V}_{\kappa} \mathcal{U}_{\kappa} \mathcal{J}_{\kappa} \frac{\partial \xi_{l}}{\partial x_{m}} n_{\xi_{l}} \mathrm{~d} \hat{\Gamma} .
\end{gathered}
$$

Finally, as will be shown later, the coupling terms are constructed to approximate

$$
\begin{aligned}
& \boldsymbol{v}_{\kappa} \mathrm{E}_{2 l \mathrm{to}(2 l-1)}^{\kappa, m} \boldsymbol{u}_{(2 l-1)} \approx \oint_{\hat{\Gamma}_{\kappa}^{(2 l-1)}} \mathcal{V}_{\kappa} \mathcal{U}_{(2 l-1)} \mathcal{J}_{\kappa} \frac{\partial \xi_{l}}{\partial x_{m}} n_{\xi_{l} \mathrm{~d}} \mathrm{~d}, \\
& \boldsymbol{v}_{\kappa} \mathrm{E}_{(2 l-1) \mathrm{to} 2 l}^{\kappa, m} \boldsymbol{u}_{2 l} \approx \oint_{\hat{\Gamma}_{\kappa}^{2 l}} \mathcal{V}_{\kappa} \mathcal{U}_{2 l} \mathcal{J}_{\kappa} \frac{\partial \xi_{l}}{\partial x_{m}} n_{\xi_{l} l} \mathrm{~d} \hat{\Gamma} .
\end{aligned}
$$

The design principle used to construct (15) is to extend the SBP property to the discretization over the full domain. To do so, the discretization must have the SBP property $\mathrm{Q}+\mathrm{Q}^{\mathrm{T}}=\mathrm{E}$ over the full domain. On interior elements, this means that the SAT that is added - which can be thought of as the difference between the numerical flux and the on element flux - must lead to a skewsymmetric operator on the on-element contributions, i.e., $\boldsymbol{u}_{\kappa}$. What is required is that the on-element contributions to the spatial derivative terms are skewsymmetric with respect to the norm matrix $\mathrm{H}$. Multiplying the on-element contributions,

$$
\frac{1}{2}\left(\mathrm{D}_{\xi_{l}} \operatorname{diag}\left(\mathcal{J} \frac{\partial \xi_{l}}{\partial x_{m}}\right)_{\kappa}+\operatorname{diag}\left(\mathcal{J} \frac{\partial \xi_{l}}{\partial x_{m}}\right)_{\kappa} \mathrm{D}_{\xi_{l}}-\mathrm{H}^{-1} \mathrm{E}_{\xi_{l}} \operatorname{diag}\left(\mathcal{J} \frac{\partial \xi_{l}}{\partial x_{m}}\right)_{\kappa}\right)
$$


Fig. 2 Depiction of the metric invariant systems that are solved: square nodes are flux nodes to which the solution is projected and at which the analytical metrics are used, while circles are the nodes at which the numerical solution is solved for.

by $\mathrm{H}$ and using the SBP property, results in

$$
\begin{aligned}
\frac{1}{2} \mathrm{~S}_{\xi_{l}} \operatorname{diag}\left(\mathcal{J} \frac{\partial \xi_{l}}{\partial x_{m}}\right)_{\kappa}-\frac{1}{4} \mathrm{E}_{\xi_{l}} \operatorname{diag}\left(\mathcal{J} \frac{\partial \xi_{l}}{\partial x_{m}}\right)_{\kappa} & \frac{1}{2} \operatorname{diag}\left(\mathcal{J} \frac{\partial \xi_{l}}{\partial x_{m}}\right)_{\kappa} \mathrm{S}_{\xi_{l}} \\
& +\frac{1}{4} \operatorname{diag}\left(\mathcal{J} \frac{\partial \xi_{l}}{\partial x_{m}}\right)_{\kappa} \mathrm{E}_{\xi_{l}},
\end{aligned}
$$

which is skew-symmetric. What remains is to construct the coupling terms, for example $\mathrm{E}_{2 l \text { to }(2 l-1)}^{\kappa, m}$, to maintain skew-symmetry.

\subsection{Construction of the coupling terms}

The construction of the coupling terms considered here borrows from the work on $h / p$-refinement in curvilinear coordinates in Ref. [18,30,29], which itself is based on the work in Crean et al. [16] on multidimensional SBP operators. The 
basic idea is to approximate the metric terms in such a way that a discrete version of the metric invariants, local to the element, is exactly satisfied (this is important in free-stream preservation and for nonlinear stability proofs). To explain the idea, consider two-dimensional SBP operators constructed on the degree 2 Gauss nodes and two elements with a shared vertical face as in Fig. 2.

The two elements interact at the flux nodes at the shared interface through the coupling terms. On these nodes, the metric terms are specified and coupling terms are created from the projection of the solution on either side using the interpolation/extrapolation $\mathrm{R}$ operators. The form of the coupling matrices, on the $\kappa^{\text {th }}$ element, are given as

$$
\begin{aligned}
& \mathrm{E}_{1 \text { to } 2}^{\kappa, m} \equiv \mathrm{R}_{\beta_{\xi_{1}}}^{\mathrm{T}} \mathrm{H}_{\xi_{1}}^{\perp} \operatorname{diag}\left(\mathcal{J} \frac{\partial \xi_{1}}{\partial x_{m}}\right)^{2} \mathrm{R}_{\alpha_{\xi_{1}}}, \mathrm{E}_{2 \text { to } 1}^{\kappa, m} \equiv-\mathrm{R}_{\alpha_{\xi_{1}}}^{\mathrm{T}} \mathrm{H}_{\xi_{1}}^{\perp} \operatorname{diag}\left(\mathcal{J} \frac{\partial \xi_{1}}{\partial x_{m}}\right)^{1} \mathrm{R}_{\beta_{\xi_{1}}}, \\
& \mathrm{E}_{3 \text { to } 4}^{\kappa, m} \equiv \mathrm{R}_{\beta_{\xi_{2}}}^{\mathrm{T}} \mathrm{H}_{\xi_{2}}^{\perp} \operatorname{diag}\left(\mathcal{J} \frac{\partial \xi_{2}}{\partial x_{m}}\right)^{4} \mathrm{R}_{\alpha_{\xi_{2}}}, \mathrm{E}_{4 \text { to } 3}^{\kappa, m} \equiv-\mathrm{R}_{\alpha_{\xi_{2}}}^{\mathrm{T}} \mathrm{H}_{\xi_{2}}^{\perp} \operatorname{diag}\left(\mathcal{J} \frac{\partial \xi_{2}}{\partial x_{m}}\right)^{3} \mathrm{R}_{\beta_{\xi_{2}}}, \\
& \mathrm{E}_{5 \text { to6 }}^{\kappa, m} \equiv \mathrm{R}_{\beta_{\xi_{3}}}^{\mathrm{T}} \mathrm{H}_{\xi_{3}}^{\perp} \operatorname{diag}\left(\mathcal{J} \frac{\partial \xi_{3}}{\partial x_{m}}\right)^{6} \mathrm{R}_{\alpha_{\xi_{3}}}, \mathrm{E}_{6 \text { to } 5}^{\kappa, m} \equiv-\mathrm{R}_{\alpha_{\xi_{3}}}^{\mathrm{T}} \mathrm{H}_{\xi_{3}}^{\perp} \operatorname{diag}\left(\mathcal{J} \frac{\partial \xi_{3}}{\partial x_{m}}\right)^{5} \mathrm{R}_{\beta_{\xi_{3}}},
\end{aligned}
$$

where the superscript on the mortar metric terms, e.g., $\operatorname{diag}\left(\mathcal{J} \frac{\partial \xi_{1}}{\partial x_{m}}\right)^{2}$ denotes the surface of the element $\kappa$ element these terms are being used for. In the coupling matrices, the metric terms are specified, for example using the analytical metrics. This means that any two elements sharing a surface have the same metric terms at the nodes of the mortar element.

Remark 2 The SAT, besides leading to stable and conservative schemes, must add design order terms; this is guaranteed by construction and the accuracy of the R operators (see Ref. [18] for a thorough discussion).

We can see that by construction, for example,

$$
\boldsymbol{v}_{\kappa}^{\mathrm{T}} \mathrm{R}_{\beta_{\xi_{1}}}^{\mathrm{T}} \mathrm{H}_{\xi_{1}}^{\perp} \operatorname{diag}\left(\mathcal{J} \frac{\partial \xi_{1}}{\partial x_{m}}\right)^{2} \mathrm{R}_{\alpha_{\xi_{1}}} \boldsymbol{u}_{1} \approx \oint_{\hat{\Gamma}_{\kappa}} \mathcal{V}_{\kappa} \mathcal{U}_{1} \mathcal{J}_{\kappa} \frac{\partial \xi_{l}}{\partial x_{m}} n_{\xi_{l}} \mathrm{~d} \hat{\Gamma}_{\kappa},
$$

since the interpolation/extrapolation operators interpolate/extrapolate $\boldsymbol{v}_{\kappa}$ and $\boldsymbol{u}_{1}$ to the nodes of the mortar element and then $\mathrm{H}_{\xi_{1}}^{\perp} \operatorname{diag}\left(\mathcal{J} \frac{\partial \xi_{1}}{\partial x_{m}}\right)^{2}$ approximates the above integral.

The first task is to show that using these coupling terms, the scheme is stable.

Theorem 1 The semi-discrete scheme (15) is stable for the periodic problem using the coupling terms (16). Therefore, using appropriate boundary SATs, the schemes are stable for nonperiodic problems.

Proof See Refs. $[18,30,29,16]$ for the required steps. 
As the interest is in the solution of conservation laws that may have discontinuous solutions, the scheme must be conservative, at least at the element level. Moreover, having a discretization that is freestream preserving is desirable. In much the same way that the metric terms for standard SBP operators need to be constructed in a particular way (see [48]) the metric terms for discretization (15) need to be computed so that a discrete version of the metric invariants (5) is satisfied; this is discussed in the following theorem:

Theorem 2 If the metric terms satisfy the following discrete form of the metric invariants (5):

$$
\begin{array}{r}
\sum_{l=1}^{3} \mathrm{D}_{\xi_{l}} \operatorname{diag}\left(\mathcal{J} \frac{\partial \xi_{l}}{\partial x_{m}}\right)_{\kappa} \mathbf{1}= \\
\mathbf{H}^{-1} \sum_{l=1}^{3}\left\{\mathrm{E}_{\xi_{l}} \operatorname{diag}\left(\mathcal{J} \frac{\partial \xi_{l}}{\partial x_{m}}\right)_{\kappa} \mathbf{1}-\left(\mathrm{E}_{2 l \mathrm{to}(2 l-1)}^{\kappa, m} \mathbf{1}+\mathrm{E}_{(2 l-1) \mathrm{to} 2 l}^{\kappa, m} \mathbf{1}\right)\right\}, \\
m=1,2,3,
\end{array}
$$

then discretization (15) is freestream preserving, element-wise conservative, and can be algebraically manipulated into the general element-wise telescoping form (11), where

$$
\begin{aligned}
& \bar{u}_{\kappa} \equiv \mathbf{1}^{\mathrm{T}} \mathrm{H} \operatorname{diag}(\mathcal{J})_{\kappa} \boldsymbol{u}_{\kappa} \\
& g_{\kappa}^{(2 l-1)} \equiv \frac{1}{2} \sum_{m=1}^{3}\left(\boldsymbol{u}_{\kappa}^{\mathrm{T}} \mathrm{E}_{2 l \text { to }(2 l-1)}^{\kappa, m} \mathbf{1}+\mathbf{1}^{\mathrm{T}} \mathrm{E}_{2 l \text { to }(2 l-1)}^{\kappa, m} \boldsymbol{u}_{2 l}\right), \\
& g_{\kappa}^{(2 l)} \equiv \frac{1}{2} \sum_{m=1}^{3}\left(\boldsymbol{u}_{\kappa}^{\mathrm{T}} \mathrm{E}_{(2 l-1) \mathrm{to} 2 l}^{\kappa, m} \mathbf{1}+\mathbf{1}^{\mathrm{T}} \mathrm{E}_{(2 l-1) \mathrm{to} 2 l}^{\kappa, m} \boldsymbol{u}_{(2 l-1)}\right) .
\end{aligned}
$$

Proof See Ref. [18] for the required steps. Note that the conditions (17) can be easily obtained by inserting the constant vector for the solution.

Construction of metric terms that satisfy (17) is addressed in Section 8 .

\section{The global SBP operator approach}

As previously stated, the guiding design principle in the SBP-framework is to extend the SBP property to the full domain discretization. In this section, this is accomplished in a direct fashion by constructing a global SBP operator over the domain and then using it to discretize (6). All SBP discretizations can be viewed in this way, i.e. that they result in global SBP operators in physical space. Here, we are constructing a global SBP operator in computational space (note that a global transformation is not assumed). The concept is best illustrated with a simple one-dimensional example with three elements. 
Consider the approximation to the derivative $\frac{\partial \mathcal{U}}{\partial \xi_{1}}$ using SBP/GSBP operators

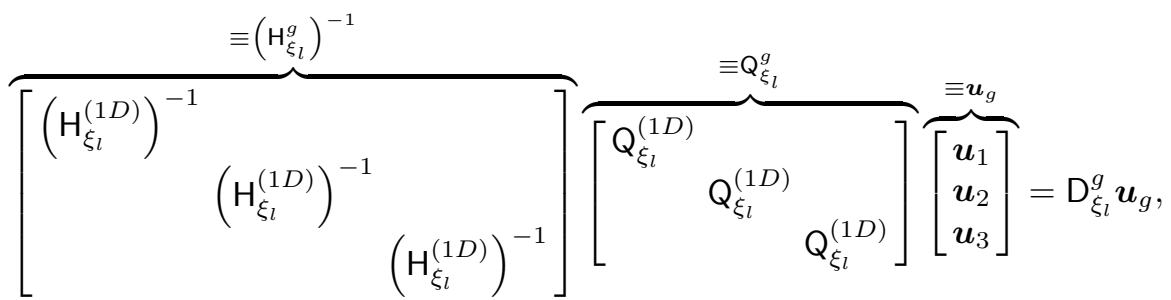

where $\mathrm{D}_{\xi_{l}}^{g} \equiv\left(\mathrm{H}_{\xi_{l}}^{g}\right)^{-1} \mathrm{Q}_{\xi_{l}}^{g}$. The resulting differentiation operator satisfies the following SBP condition:

$$
\mathrm{Q}_{\xi_{l}}^{g}+\left(\mathrm{Q}_{\xi_{l}}^{g}\right)^{\mathrm{T}}=\mathrm{E}_{\xi_{l}}^{g} \equiv\left[\begin{array}{lll}
\mathrm{E}_{\xi_{l}}^{(1 D)} & & \\
& \mathrm{E}_{\xi_{l}}^{(1 D)} & \\
& & \mathrm{E}_{\xi_{l}}^{(1 D)}
\end{array}\right] .
$$

While $\mathrm{E}_{\xi_{1}}^{g}$ satisfies the polynomial exactness conditions on $\mathrm{E}$, it does not have an outer product expansion. This can be remedied by using symmetric SATs to couple the interfaces of the three elements as follows:

$$
\begin{aligned}
& \mathbf{H}_{\xi_{l}}^{g} \equiv\left[\begin{array}{lll}
\mathbf{H}_{\xi_{l}}^{(1 D)} & & \\
& \mathbf{H}_{\xi_{l}}^{(1 D)} & \\
& & \mathbf{H}_{\xi_{l}}^{(1 D)}
\end{array}\right],
\end{aligned}
$$

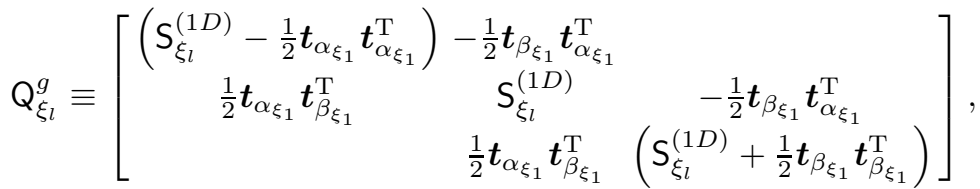

$$
\begin{aligned}
& \mathrm{S}_{\xi_{l}}^{g} \equiv\left[\begin{array}{ccc}
\mathrm{S}_{\xi_{l}}^{(1 D)} & -\frac{1}{2} \boldsymbol{t}_{\beta_{\xi_{1}}} \boldsymbol{t}_{\alpha_{\xi_{1}}}^{\mathrm{T}} & \\
\frac{1}{2} \boldsymbol{t}_{\alpha_{\xi_{1}}} \boldsymbol{t}_{\beta_{\xi_{1}}}^{\mathrm{T}} & \mathrm{S}_{\xi_{l}}^{(1 D)} & -\frac{1}{2} \boldsymbol{t}_{\beta_{\xi_{1}}} \boldsymbol{t}_{\alpha_{\xi_{1}}}^{\mathrm{T}} \\
& \frac{1}{2} \boldsymbol{t}_{\alpha_{\xi_{1}}} \boldsymbol{t}_{\beta_{\xi_{1}}}^{\mathrm{T}} & \mathrm{S}_{\xi_{l}}^{(1 D)}
\end{array}\right]
\end{aligned}
$$

and $\mathrm{E}_{\xi_{l}}^{g} \equiv \mathrm{E}_{\alpha_{\xi_{1}}}^{g}+\mathrm{E}_{\beta_{\xi_{1}}}^{g}$, where

$$
\begin{gathered}
\mathrm{E}_{\alpha_{\xi_{1}}}^{g} \equiv \boldsymbol{t}_{\alpha_{\xi_{1}}}^{g}\left(\boldsymbol{t}_{\alpha_{\xi_{1}}}^{g}\right)^{\mathrm{T}}=-\left[\begin{array}{ccc}
\boldsymbol{t}_{\alpha_{\xi_{1}}} \boldsymbol{t}_{\alpha_{\xi_{1}}}^{\mathrm{T}} & 0 & 0 \\
0 & 0 & 0 \\
0 & 0 & 0
\end{array}\right], \quad \mathrm{E}_{\beta_{\xi_{1}}}^{g} \equiv \boldsymbol{t}_{\beta_{\xi_{1}}}^{g}\left(\boldsymbol{t}_{\beta_{\xi_{1}}}^{g}\right)^{\mathrm{T}}=\left[\begin{array}{lll}
0 & 0 & 0 \\
0 & 0 & 0 \\
0 & 0 & \boldsymbol{t}_{\beta_{\xi_{1}}} \boldsymbol{t}_{\beta_{\xi_{1}}}^{\mathrm{T}}
\end{array}\right], \\
\boldsymbol{t}_{\alpha_{\xi_{1}}}^{g}=\left[\boldsymbol{t}_{\alpha_{\xi_{1}}}^{\mathrm{T}}, \mathbf{0}^{\mathrm{T}}, \mathbf{0}^{\mathrm{T}}\right]^{\mathrm{T}}, \quad \boldsymbol{t}_{\beta_{\xi_{1}}}^{g}=\left[\mathbf{0}^{\mathrm{T}}, \mathbf{0}^{\mathrm{T}}, \boldsymbol{t}_{\beta_{\xi_{1}}}^{\mathrm{T}}\right]^{\mathrm{T}} .
\end{gathered}
$$

The resulting global SBP operator has the desired property of $\mathrm{Q}_{\xi_{l}}^{g}+\left(\mathrm{Q}_{\xi_{l}}^{g}\right)^{\mathrm{T}}=$ $\mathrm{E}_{\xi_{l}}^{g}$. 
The global operator approach allows the standard finite-difference approach for the approximation of the metric terms to be reused. We note that the boundary conditions are imposed using SATs. The discretization for the periodic problem has the form

$\operatorname{diag}(\mathcal{J})_{g} \frac{\mathrm{d} \boldsymbol{u}_{g}}{\mathrm{~d} t}+\frac{1}{2} \mathrm{H}_{g}^{-1} \sum_{l, m=1}^{3}\left(\mathrm{Q}_{\xi_{l}}^{g} \operatorname{diag}\left(\mathcal{J} \frac{\partial \xi_{l}}{\partial x_{m}}\right)_{g}+\operatorname{diag}\left(\mathcal{J} \frac{\partial \xi_{l}}{\partial x_{m}}\right)_{g} \mathbf{Q}_{\xi_{l}}^{g}\right) \boldsymbol{u}_{g}=\mathbf{0}$,

where $\mathrm{Q}_{\xi_{l}}^{g}$ has been appropriately constructed to absorb the periodic boundary conditions and is therefore different than in the above example at the boundaries. form

On the $\kappa^{\text {th }}$ element, the global SBP operator $\mathrm{D}_{\xi_{l}}^{g} \boldsymbol{u}_{g} \equiv \mathrm{H}_{g}^{-1} \mathrm{Q}_{\xi_{l}}^{g} \boldsymbol{u}_{g}$ has the

$$
\begin{aligned}
& \left(\mathrm{D}_{\xi_{l}}^{g} \boldsymbol{u}_{g}\right)((\kappa-1) N+1: \kappa N)= \\
& \quad \mathrm{H}^{-1}\left(\mathrm{Q}_{\xi_{l}} \boldsymbol{u}_{\kappa}-\frac{1}{2} \mathrm{E}_{\xi_{l}} \boldsymbol{u}_{\kappa}+\frac{1}{2} \mathrm{R}_{\beta_{\xi_{l}}}^{\mathrm{T}} \mathrm{H}_{\xi_{l}}^{\perp} \mathrm{R}_{\alpha_{\xi_{l}}} \boldsymbol{u}_{2 l}-\frac{1}{2} \mathrm{R}_{\alpha_{\xi_{l}}}^{\mathrm{T}} \mathrm{H}_{\xi_{l}}^{\perp} \mathrm{R}_{\beta_{\xi_{l}}} \boldsymbol{u}_{(2 l-1)}\right),
\end{aligned}
$$

where Matlab notation has been used and $N$ is the total number of nodes in the element; thus, on the $\kappa^{\text {th }}$ element, the discretization of (6) using the global SBP operator approach is given as

$$
\begin{aligned}
& \operatorname{diag}(\mathcal{J})_{\kappa} \frac{\mathrm{d} \boldsymbol{u}_{\kappa}}{\mathrm{d} t} \\
& +\frac{1}{2} \mathrm{H}^{-1} \sum_{l, m=1}^{3}\left(\mathrm{Q}_{\xi_{l}} \operatorname{diag}\left(\mathcal{J} \frac{\partial \xi_{l}}{\partial x_{m}}\right)_{\kappa}+\operatorname{diag}\left(\mathcal{J} \frac{\partial \xi_{l}}{\partial x_{m}}\right)_{\kappa} \mathbf{Q}_{\xi_{l}}\right) \boldsymbol{u}_{\kappa}= \\
& \frac{1}{4} \mathrm{H}^{-1} \sum_{l, m=1}^{3}\left(\operatorname{diag}\left(\mathcal{J} \frac{\partial \xi_{l}}{\partial x_{m}}\right)_{\kappa} \mathrm{E}_{\xi_{l}}+\mathrm{E}_{\xi_{l}} \operatorname{diag}\left(\mathcal{J} \frac{\partial \xi_{l}}{\partial x_{m}}\right)_{\kappa}\right) \boldsymbol{u}_{\kappa} \\
& -\frac{1}{4} \mathrm{H}^{-1} \sum_{l, m=1}^{3}\left(\mathrm{R}_{\beta_{\xi_{l}}}^{\mathrm{T}} \mathrm{H}_{\xi_{l}}^{\perp} \mathrm{R}_{\alpha_{\xi_{l}}} \operatorname{diag}\left(\mathcal{J} \frac{\partial \xi_{l}}{\partial x_{m}}\right)^{2 l} \boldsymbol{u}_{2 l}\right. \\
& \left.-\mathrm{R}_{\alpha_{\xi_{l}}}^{\mathrm{T}} \mathrm{H}_{\xi_{l}}^{\perp} \mathrm{R}_{\beta_{\xi_{l}}} \operatorname{diag}\left(\mathcal{J} \frac{\partial \xi_{l}}{\partial x_{m}}\right)^{(2 l-1)} \boldsymbol{u}_{(2 l-1)}\right) \\
& -\frac{1}{4} \mathrm{H}^{-1} \sum_{l, m=1}^{3}\left(\operatorname{diag}\left(\mathcal{J} \frac{\partial \xi_{l}}{\partial x_{m}}\right)_{\kappa} \mathrm{R}_{\beta_{\xi_{l}}}^{\mathrm{T}} \mathrm{H}_{\xi_{l}}^{\perp} \mathrm{R}_{\alpha_{\xi_{l}}} \boldsymbol{u}_{2 l}\right. \\
& \left.-\operatorname{diag}\left(\mathcal{J} \frac{\partial \xi_{l}}{\partial x_{m}}\right)_{\kappa} \mathrm{R}_{\alpha_{\xi_{l}}}^{\mathrm{T}} \mathrm{H}_{\xi_{l}}^{\perp} \mathrm{R}_{\beta_{\xi_{l}}} \boldsymbol{u}_{(2 l-1)}\right) \text {. }
\end{aligned}
$$

While conceptually we have constructed an SBP operator over the entire mesh, its use does not require a global mapping. The next theorem proves that the scheme is design order for general meshes that satisfy Assumption 1. 
1 Theorem 3 The discretization (20) is design order for general meshes satisfying Assumption 20.

Proof The volume terms are design-order for diagonal norms since

$$
\begin{aligned}
& \frac{1}{2} \mathrm{H}^{-1} \sum_{l, m=1}^{3}\left(\mathrm{Q}_{\xi_{l}} \operatorname{diag}\left(\mathcal{J} \frac{\partial \xi_{l}}{\partial x_{m}}\right)_{\kappa}+\operatorname{diag}\left(\mathcal{J} \frac{\partial \xi_{l}}{\partial x_{m}}\right)_{\kappa} \mathrm{Q}_{\xi_{l}}\right) \boldsymbol{u}_{\kappa}= \\
& \frac{1}{2} \sum_{l, m=1}^{3}\left(\mathrm{D}_{\xi_{l}} \operatorname{diag}\left(\mathcal{J} \frac{\partial \xi_{l}}{\partial x_{m}}\right)_{\kappa}+\operatorname{diag}\left(\mathcal{J} \frac{\partial \xi_{l}}{\partial x_{m}}\right)_{\kappa} \mathrm{D}_{\xi_{l}}\right) \boldsymbol{u}_{\kappa} .
\end{aligned}
$$

Next, we split the SATs into two sets two of terms; the first is given as

$$
\begin{aligned}
\boldsymbol{S} \boldsymbol{A} \boldsymbol{T}_{1} \equiv \frac{1}{4} \mathrm{H}^{-1} \sum_{l, m=1}^{3} & \left(\operatorname{diag}\left(\mathcal{J} \frac{\partial \xi_{l}}{\partial x_{m}}\right)_{\kappa} \mathrm{E}_{\xi_{l}} \boldsymbol{u}_{\kappa}-\operatorname{diag}\left(\mathcal{J} \frac{\partial \xi_{l}}{\partial x_{m}}\right)_{\kappa} \mathrm{R}_{\beta_{\xi_{l}}}^{\mathrm{T}} \mathrm{H}_{\xi_{l}}^{\perp} \mathrm{R}_{\alpha_{\xi_{l}}} \boldsymbol{u}_{2 l}\right. \\
& \left.+\operatorname{diag}\left(\mathcal{J} \frac{\partial \xi_{l}}{\partial x_{m}}\right)_{\kappa} \mathrm{R}_{\alpha_{\xi_{l}}}^{\mathrm{T}} \mathrm{H}_{\xi_{l}}^{\perp} \mathrm{R}_{\beta_{\xi_{l}}} \boldsymbol{u}_{(2 l-1)}\right) .
\end{aligned}
$$

Using the decomposition $\mathrm{E}_{\xi_{l}}=\mathrm{R}_{\beta_{\xi_{l}}}^{\mathrm{T}} \mathrm{H}_{\xi_{l}}^{\perp} \mathrm{R}_{\beta_{\xi_{l}}}-\mathrm{R}_{\alpha_{\xi_{l}}}^{\mathrm{T}} \mathrm{H}_{\xi_{l}}^{\perp} \mathrm{R}_{\alpha_{\xi_{l}}}$ and grouping terms results in

$$
\begin{aligned}
\boldsymbol{S} \boldsymbol{A} \boldsymbol{T}_{1}=\frac{1}{4} \mathrm{H}^{-1} \sum_{l, m=1}^{3} & \left\{\operatorname{diag}\left(\mathcal{J} \frac{\partial \xi_{l}}{\partial x_{m}}\right)_{\kappa} \mathrm{R}_{\beta_{\xi_{l}}^{\mathrm{T}}}^{\mathrm{T}} \mathrm{H}_{\xi_{l}}^{\perp}\left(\mathrm{R}_{\beta_{\xi_{l}}} \boldsymbol{u}_{\kappa}-\mathrm{R}_{\alpha_{\xi_{l}}} \boldsymbol{u}_{2 l}\right)\right. \\
& \left.-\operatorname{diag}\left(\mathcal{J} \frac{\partial \xi_{l}}{\partial x_{m}}\right)_{\kappa} \mathrm{R}_{\alpha_{\xi_{l}}}^{\mathrm{T}} \mathrm{H}_{\xi_{l}}^{\perp}\left(\mathrm{R}_{\alpha_{\xi_{l}}} \boldsymbol{u}_{\kappa}-\mathrm{R}_{\beta_{\xi_{l}}} \boldsymbol{u}_{(2 l-1)}\right)\right\} .
\end{aligned}
$$

The action of the interpolation/extrapolation operators, $\mathrm{R}$, is to interpolate/extrapolate quantities to the same nodes on the mortar element and it is clear that the above is design order close to zero.

The second set of terms in the SAT are given as

$$
\begin{aligned}
\boldsymbol{S} \boldsymbol{A} \boldsymbol{T}_{2} \equiv \frac{1}{4} \mathrm{H}^{-1} \sum_{l, m=1}^{3} & \left(\mathrm{E}_{\xi_{l}} \operatorname{diag}\left(\mathcal{J} \frac{\partial \xi_{l}}{\partial x_{m}}\right)_{\kappa} \boldsymbol{u}_{\kappa}-\mathrm{R}_{\beta_{\xi_{l}}}^{\mathrm{T}} \mathrm{H}_{\xi_{l}}^{\perp} \mathrm{R}_{\alpha_{\xi_{l}}} \operatorname{diag}\left(\mathcal{J} \frac{\partial \xi_{l}}{\partial x_{m}}\right)^{2 l} \boldsymbol{u}_{2 l}\right. \\
& \left.+\mathrm{R}_{\alpha_{\xi_{l}}}^{\mathrm{T}} \mathrm{H}_{\xi_{l}}^{\perp} \mathrm{R}_{\beta_{\xi_{l}}} \operatorname{diag}\left(\mathcal{J} \frac{\partial \xi_{l}}{\partial x_{m}}\right)^{(2 l-1)} \boldsymbol{u}_{(2 l-1)}\right),
\end{aligned}
$$

and an identical analysis shows that this too is design order.

Using (19) and the global SBP property it is straightforward to prove stability:

Theorem 4 Discretization (19) is stable for the periodic problem and therefore, with appropriate SATs for the weak imposition of boundary conditions, stable for the nonperiodic problem. 
$\underline{40}$

41

$\underline{42}$

$\frac{43}{44}$

$\frac{44}{45}$

$\underline{46}$ $\underline{47}$
Proof Multiplying (19) by $\boldsymbol{u}_{g}^{\mathrm{T}} \mathrm{H}_{g}$ gives

$$
\begin{aligned}
\boldsymbol{u}_{g}^{\mathrm{T}} \mathrm{H}_{g} & \operatorname{diag}(\mathcal{J})_{g} \frac{\mathrm{d} \boldsymbol{u}_{g}}{\mathrm{~d} t} \\
& +\frac{1}{2} \boldsymbol{u}_{g}^{\mathrm{T}} \sum_{l, m=1}^{3}\left(\mathrm{Q}_{\xi_{l}}^{g} \operatorname{diag}\left(\mathcal{J} \frac{\partial \xi_{l}}{\partial x_{m}}\right)_{g}+\operatorname{diag}\left(\mathcal{J} \frac{\partial \xi_{l}}{\partial x_{m}}\right)_{g} \mathrm{Q}_{\xi_{l}}^{g}\right) \boldsymbol{u}_{g}=\mathbf{0} .
\end{aligned}
$$

Since $\mathrm{Q}_{\xi_{l}}^{g} \operatorname{diag}\left(\mathcal{J} \frac{\partial \xi_{l}}{\partial x_{m}}\right)_{g}+\operatorname{diag}\left(\mathcal{J} \frac{\partial \xi_{l}}{\partial x_{m}}\right)_{g} \mathbf{Q}_{\xi_{l}}^{g}$ is skew symmetric, this implies that

$$
\boldsymbol{u}_{g}^{\mathrm{T}} \mathrm{H}_{g} \operatorname{diag}(\mathcal{J})_{g} \frac{\mathrm{d} \boldsymbol{u}_{g}}{\mathrm{~d} t}=0,
$$

which demonstrates that the scheme is stable.

The conditions on freestream preservation and element-wise conservation are easily derived.

Theorem 5 If the metric terms satisfy the following discrete form of the metric invariants (5):

$$
\sum_{l=1}^{3} \mathrm{D}_{\xi_{l}}^{g} \operatorname{diag}\left(\mathcal{J} \frac{\partial \xi_{l}}{\partial x_{m}}\right)_{g} \mathbf{1}_{g}=\mathbf{0}, \quad m=1,2,3
$$

then discretization (19) is freestream preserving, element-wise conservative, and can be algebraically manipulated into the general telescoping flux form (11), where

$\bar{u}_{\kappa} \equiv \mathbf{1}^{\mathrm{T}} \mathrm{H} \operatorname{diag}(\mathcal{J})_{\kappa} \boldsymbol{u}_{\kappa}$,

$g_{\kappa}^{(2 l)}=$

$\frac{\mathbf{1}^{\mathrm{T}}}{4} \sum_{m=1}^{3}\left(\mathrm{R}_{\beta_{\xi_{l}}^{\mathrm{T}}}^{\mathrm{T}} \mathrm{H}_{\xi_{l}}^{\perp} \mathrm{R}_{\beta_{\xi_{l}}} \operatorname{diag}\left(\mathcal{J} \frac{\partial \xi_{l}}{\partial x_{m}}\right)_{\kappa} \boldsymbol{u}_{\kappa}+\operatorname{diag}\left(\mathcal{J} \frac{\partial \xi_{l}}{\partial x_{m}}\right)_{\kappa} \mathrm{R}_{\beta_{\xi_{l}}}^{\mathrm{T}} \mathrm{H}_{\xi_{l}}^{\perp} \mathrm{R}_{\alpha_{\xi_{l}}} \boldsymbol{u}_{2 l}\right.$

$\left.+\operatorname{diag}\left(\mathcal{J} \frac{\partial \xi_{l}}{\partial x_{m}}\right)^{2 l} \mathrm{R}_{\alpha_{\xi_{l}}}^{\mathrm{T}} \mathrm{H}_{\xi_{l}}^{\perp} \mathrm{R}_{\beta_{\xi_{l}}} \boldsymbol{u}_{\kappa}+\mathrm{R}_{\beta_{\xi_{l}}}^{\mathrm{T}} \mathrm{H}_{\xi_{l}}^{\perp} \mathrm{R}_{\alpha_{\xi_{l}}} \operatorname{diag}\left(\mathcal{J} \frac{\partial \xi_{l}}{\partial x_{m}}\right)^{2 l} \boldsymbol{u}_{2 l}\right)$,

$g_{\kappa}^{(2 l-1)}=$

$-\frac{\mathbf{1}^{\mathrm{T}}}{4} \sum_{m=1}^{3}\left(\mathrm{R}_{\alpha_{\xi_{l}}}^{\mathrm{T}} \mathrm{H}_{\xi_{l}}^{\perp} \mathrm{R}_{\alpha_{\xi_{l}}} \operatorname{diag}\left(\mathcal{J} \frac{\partial \xi_{l}}{\partial x_{m}}\right)_{\kappa} \boldsymbol{u}_{\kappa}\right.$

$+\operatorname{diag}\left(\mathcal{J} \frac{\partial \xi_{l}}{\partial x_{m}}\right)_{\kappa} \mathrm{R}_{\alpha_{\xi_{l}}}^{\mathrm{T}} \mathrm{H}_{\xi_{l}}^{\perp} \mathrm{R}_{\beta_{\xi_{l}}} \boldsymbol{u}_{(2 l-1)}+\operatorname{diag}\left(\mathcal{J} \frac{\partial \xi_{l}}{\partial x_{m}}\right)^{(2 l-1)} \mathrm{R}_{\beta_{\xi_{l}}}^{\mathrm{T}} \mathrm{H}_{\xi_{l}}^{\perp} \mathrm{R}_{\alpha_{\xi_{l}}} \boldsymbol{u}_{\kappa}$

$\left.+\mathrm{R}_{\alpha_{\xi_{l}}}^{\mathrm{T}} \mathrm{H}_{\xi_{l}}^{\perp} \mathrm{R}_{\beta_{\xi_{l}}} \operatorname{diag}\left(\mathcal{J} \frac{\partial \xi_{l}}{\partial x_{m}}\right)^{(2 l-1)} \boldsymbol{u}_{(2 l-1)}\right)$.

Proof The steps are similar to those in Refs. [19,18]. 


\section{Approximation of the metric terms}

This section covers how to approximate the metric terms for the mortar element approach and the global SBP operator approach. In both cases, an approximation to the metric terms is sought that is accurate and satisfies the discrete versions of the metric invariants (5), which is necessary for elementwise conservation (see Theorems 2 and 5 ).

8.1 Metric terms in the mortar element approach

The solution of (17) is now described; the approach is identical to that in Crean et al. [16].

Assumption 2 For the mortar-element approach, it is assumed that access to a polynomial, tensor-product, curvilinear coordinate transformation local to each element is available. In the numerical examples, this assumption is satisfied by using B-splines.

The entries of the diagonal matrices diag $\left(\mathcal{J} \frac{\partial \xi_{l}}{\partial x_{m}}\right)_{\kappa}$ are determined by solving a strictly convex quadratic optimization problem:

$\min _{\boldsymbol{a}_{m}^{\kappa}} \frac{1}{2}\left(\boldsymbol{a}_{m}^{\kappa}-\boldsymbol{a}_{m, \text { target }}^{\kappa}\right)^{\mathrm{T}}\left(\boldsymbol{a}_{m}^{\kappa}-\boldsymbol{a}_{m, \text { target }}^{\kappa}\right)$, subject to $\mathrm{M} \boldsymbol{a}_{m}^{\kappa}=\boldsymbol{c}_{m}^{\kappa}, m=1,2,3$,

where

$$
\left(\boldsymbol{a}_{m}^{\kappa}\right)^{\mathrm{T}} \equiv \mathbf{1}^{\mathrm{T}}\left[\operatorname{diag}\left(\mathcal{J} \frac{\partial \xi_{1}}{\partial x_{m}}\right)_{\kappa}, \operatorname{diag}\left(\mathcal{J} \frac{\partial \xi_{2}}{\partial x_{m}}\right)_{\kappa}, \operatorname{diag}\left(\mathcal{J} \frac{\partial \xi_{3}}{\partial x_{m}}\right)_{\kappa}\right] .
$$

The system of equations $\mathbf{M} \boldsymbol{a}_{m}^{\kappa}=\boldsymbol{c}_{m}^{\kappa}$ is a rearranged version of the discrete metric invariant conditions which is reproduced here. For (17) the following is used,

$$
\sum_{l=1}^{3} \mathrm{Q}_{\xi_{l}}^{\mathrm{T}} \operatorname{diag}\left(\mathcal{J} \frac{\partial \xi_{l}}{\partial x_{m}}\right)_{\kappa} \mathbf{1}=\sum_{l=1}^{3}\left(\mathrm{E}_{2 l \mathrm{to}(2 l-1)}^{\kappa, m} \mathbf{1}+\mathrm{E}_{(2 l-1) \mathrm{to} 2 l}^{\kappa, m} \mathbf{1}\right), \quad m=1,2,3,
$$

the system matrix, $M$, is

$$
\mathrm{M} \equiv\left[\mathrm{Q}_{\xi_{1}}^{\mathrm{T}}, \mathrm{Q}_{\xi_{2}}^{\mathrm{T}}, \mathrm{Q}_{\xi_{3}}^{\mathrm{T}}\right]
$$

and $(22)$

$$
\boldsymbol{c}_{m}^{\kappa} \equiv \sum_{l=1}^{3}\left(\mathrm{E}_{2 l \mathrm{to}(2 l-1)}^{\kappa, m} \mathbf{1}+\mathrm{E}_{(2 l-1) \mathrm{to} 2 l}^{\kappa, m} \mathbf{1}\right) .
$$

The target value, $\boldsymbol{a}_{m \text {,target }}^{\kappa}$ is taken as the analytical metrics. 
An optimal solution in the Cartesian 2-norm is given by (for more details see Proposition 1 in Crean et al. [16])

$$
\boldsymbol{a}_{m}^{\kappa}=\boldsymbol{a}_{m, \text { target }}^{\kappa}-(\mathrm{M})^{\dagger}\left(\mathrm{M} \boldsymbol{a}_{m, \text { target }}^{\kappa}-\boldsymbol{c}_{m}^{\kappa}\right) \text {. }
$$

The Moore-Penrose pseudo inverse of $\mathrm{M},(\mathrm{M})^{\dagger}$, is computed from the singular value decomposition of $\mathrm{M}$ as given by

$$
\mathrm{M}=\mathrm{U} \Sigma \mathrm{V}^{\mathrm{T}}, \quad(\mathrm{M})^{\dagger}=\mathrm{V} \Sigma^{\dagger} \mathrm{U} .
$$

The matrix $\mathrm{U}$ is a $N \times N$ unitary matrix, the matrix $\Sigma$ contains the singular values along its diagonal, and $\mathrm{V}$ is a $3 N \times N$ matrix with orthonormal columns. The matrix $\mathrm{M}$ has row rank of $N-1$ and thus one zero singular value. Therefore, the $\Sigma^{\dagger}$ is computed as

$$
\Sigma^{\dagger}(i, i)=\frac{1}{\Sigma(i, i)}, \quad i=2,3, \ldots, N .
$$

Furthermore, in order to solve the metric invariant system, $\boldsymbol{c}_{m}^{\kappa}$ must be in the null space of $U_{\kappa}$. The singular vector that spans this null space is the constant vector and therefore this means that $\mathbf{1}_{\kappa}^{\mathrm{T}} \boldsymbol{c}_{m}^{\kappa}=0$. This is guaranteed if the metric terms are constructed from degree $p$ tensor-product curvilinear coordinate transformations, since the $\mathrm{E}$ matrices are at least degree $2 p-1$. To see this, consider integrating the metric invariants over the domain

$$
\int_{\hat{\Omega}_{\kappa}} \sum_{l=1}^{3} \frac{\partial}{\partial \xi_{l}}\left(\mathcal{J}_{\kappa} \frac{\partial \xi_{l}}{\partial x_{m}}\right) \mathrm{d} \hat{\Omega}=\oint_{\hat{\Gamma}_{\kappa}} \sum_{l=1}^{3}\left(\mathcal{J}_{\kappa} \frac{\partial \xi_{l}}{\partial x_{m}}\right) n_{\xi_{l}} \mathrm{~d} \hat{\Gamma}=0, \quad m=1,2,3,
$$

where the first equality results from integration by parts. The condition $\mathbf{1}_{\kappa}^{\mathrm{T}} \boldsymbol{c}_{m}^{\kappa}=$ 0 is the discrete equivalent to the analytical surface integral condition. Thus, if the surface integrals in $\mathbf{1}_{\kappa}^{\mathrm{T}} \boldsymbol{c}_{m}^{\kappa}$ are exact, this condition is met. For a degree $p$ tensor-product curvilinear coordinate transformation, the metric terms are of degree $2 p-1$, and since the surface mass matrix is of degree $2 p-1$, the surface integrations are exact.

The procedure proposed in this section results in metric terms that are as close as possible to the analytical metric terms while satisfying the discrete metric invariant conditions. As a result, it can be seen that the LHS of the discrete metric invariant conditions is a consistent discretization of the continuous metric invariant conditions (5). Moreover, the RHS term results in a pointwise difference between the analytical and approximated metrics that is design order.

Remark 3 For two-dimensional problems with tensor-product degree $p$ curvilinear coordinate transformations, the optimization procedure described above is not necessary because the discrete metric invariants are satisfied by the evaluation of the analytical metrics on the mesh. However, for three-dimensional problems this approach is necessary. If the volume metric terms are approximated using a standard approach such as that of Thomas and Lombard and the metrics on the mortar are specified either using a standard approach or analytical metrics, the resulting discretization will fail to preserve a freestream. 
8.2 Metric terms in the global SBP operator approach

The global SBP operators are used to approximate the metric terms that arise from the curvilinear coordinate transformation. There are a number of different ways of approximating the metric terms so that the discrete metric invariants are satisfied (see Refs. [22,23,47,48]; for example, using the Thomas and Lombard [47] approach, the metric terms are approximated as

$$
\begin{aligned}
& \mathcal{J}\left(\boldsymbol{\xi}_{\mathbf{1}}\right)_{x} \approx \mathrm{D}_{\xi_{3}}^{g}\left(\operatorname{diag}\left(\boldsymbol{x}_{3}\right) \mathrm{D}_{\xi_{2}}^{g} \boldsymbol{x}_{2}\right)-\mathrm{D}_{\xi_{2}}^{g}\left(\operatorname{diag}\left(\boldsymbol{x}_{3}\right) \mathrm{D}_{\xi_{3}}^{g} \boldsymbol{x}_{2}\right), \\
& \mathcal{J}\left(\boldsymbol{\xi}_{\mathbf{1}}\right)_{y} \approx \mathrm{D}_{\xi_{3}}^{g}\left(\operatorname{diag}\left(\boldsymbol{x}_{1}\right) \mathrm{D}_{\xi_{2}}^{g} \boldsymbol{x}_{3}\right)-\mathrm{D}_{\xi_{2}}^{g}\left(\operatorname{diag}\left(\boldsymbol{x}_{1}\right) \mathrm{D}_{\xi_{3}}^{g} \boldsymbol{x}_{3}\right), \\
& \mathcal{J}\left(\boldsymbol{\xi}_{\mathbf{1}}\right)_{z} \approx \mathrm{D}_{\xi_{3}}^{g}\left(\operatorname{diag}\left(\boldsymbol{x}_{2}\right) \mathrm{D}_{\xi_{2}}^{g} \boldsymbol{x}_{1}\right)-\mathrm{D}_{\xi_{2}}^{g}\left(\operatorname{diag}\left(\boldsymbol{x}_{2}\right) \mathrm{D}_{\xi_{3}}^{g} \boldsymbol{x}_{1}\right), \\
& \mathcal{J}\left(\boldsymbol{\xi}_{\mathbf{2}}\right)_{x} \approx \mathrm{D}_{\xi_{1}}^{g}\left(\operatorname{diag}\left(\boldsymbol{x}_{3}\right) \mathrm{D}_{\xi_{3}}^{g} \boldsymbol{x}_{2}\right)-\mathrm{D}_{\xi_{3}}^{g}\left(\operatorname{diag}\left(\boldsymbol{x}_{3}\right) \mathrm{D}_{\xi_{1}}^{g} \boldsymbol{x}_{2}\right), \\
& \mathcal{J}\left(\boldsymbol{\xi}_{\mathbf{2}}\right)_{y} \approx \mathrm{D}_{\xi_{1}}^{g}\left(\operatorname{diag}\left(\boldsymbol{x}_{1}\right) \mathrm{D}_{\xi_{3}}^{g} \boldsymbol{x}_{3}\right)-\mathrm{D}_{\xi_{3}}^{g}\left(\operatorname{diag}\left(\boldsymbol{x}_{1}\right) \mathrm{D}_{\xi_{1}}^{g} \boldsymbol{x}_{3}\right), \\
& \mathcal{J}\left(\boldsymbol{\xi}_{\mathbf{2}}\right)_{z} \approx \mathrm{D}_{\xi_{1}}^{g}\left(\operatorname{diag}\left(\boldsymbol{x}_{2}\right) \mathrm{D}_{\xi_{3}}^{g} \boldsymbol{x}_{1}\right)-\mathrm{D}_{\xi_{3}}^{g}\left(\operatorname{diag}\left(\boldsymbol{x}_{2}\right) \mathrm{D}_{\xi_{1}}^{g} \boldsymbol{x}_{1}\right), \\
& \mathcal{J}\left(\boldsymbol{\xi}_{\mathbf{3}}\right)_{x} \approx \mathrm{D}_{\xi_{2}}^{g}\left(\operatorname{diag}\left(\boldsymbol{x}_{3}\right) \mathrm{D}_{\xi_{1}}^{g} \boldsymbol{x}_{2}\right)-\mathrm{D}_{\xi_{1}}^{g}\left(\operatorname{diag}\left(\boldsymbol{x}_{3}\right) \mathrm{D}_{\xi_{2}}^{g} \boldsymbol{x}_{2}\right), \\
& \mathcal{J}\left(\boldsymbol{\xi}_{\mathbf{3}}\right)_{y} \approx \mathrm{D}_{\xi_{2}}^{g}\left(\operatorname{diag}\left(\boldsymbol{x}_{1}\right) \mathrm{D}_{\xi_{1}}^{g} \boldsymbol{x}_{3}\right)-\mathrm{D}_{\xi_{1}}^{g}\left(\operatorname{diag}\left(\boldsymbol{x}_{1}\right) \mathrm{D}_{\xi_{2}}^{g} \boldsymbol{x}_{3}\right), \\
& \mathcal{J}\left(\boldsymbol{\xi}_{\mathbf{3}}\right)_{z} \approx \mathrm{D}_{\xi_{2}}^{g}\left(\operatorname{diag}\left(\boldsymbol{x}_{2}\right) \mathrm{D}_{\xi_{1}}^{g} \boldsymbol{x}_{1}\right)-\mathrm{D}_{\xi_{1}}^{g}\left(\operatorname{diag}\left(\boldsymbol{x}_{2}\right) \mathrm{D}_{\xi_{2}}^{g} \boldsymbol{x}_{1}\right),
\end{aligned}
$$

where the notation, for example $\mathcal{J}\left(\boldsymbol{\xi}_{1}\right)_{x}$, is the vector constructed by evaluating $\mathcal{J} \frac{\partial \xi_{1}}{\partial x}$ at the mesh nodes.

As long as the approximated metrics satisfy the discrete metric invariants at the volume nodes of each element individually, then the following result holds [2]:

Theorem 6 If the metric terms are approximated so that the discrete metric invariants are satisfied at the volume nodes, for example using (24), and the outer derivative of the discrete metric invariants is approximated using global SBP operators (for example, the underlined terms in $\mathcal{J}\left(\boldsymbol{\xi}_{\mathbf{1}}\right)_{x} \approx \underline{\mathrm{D}_{\xi_{3}}^{g}}\left(\operatorname{diag}\left(\boldsymbol{x}_{3}\right) \mathrm{D}_{\xi_{2}}^{g} \boldsymbol{x}_{2}\right)-$ $\left.\mathrm{D}_{\xi_{2}}^{g}\left(\operatorname{diag}\left(\boldsymbol{x}_{3}\right) \mathrm{D}_{\xi_{3}}^{g} \boldsymbol{x}_{2}\right)\right)$, then, the discrete metric invariants are satisfied, i.e.,

$$
\sum_{l=1}^{3} \mathrm{D}_{\xi_{l}}^{g} \operatorname{diag}\left(\mathcal{J} \frac{\partial \xi_{l}}{\partial x_{m}}\right)_{\kappa}^{g} \mathbf{1}_{g}=0
$$

Proof The proof follows immediately via the form of the approximation to the metric terms and the commutative property of the global SBP operators. 
Remark 4 While for tensor-product global SBP operators, we can use standard approaches for the approximation of the metric terms, the extension of these operators to multidimensional SBP operators requires the metric approximation approach presented for the mortar-element approach, because the SBP operators no longer commute.

\section{Discretizations with diagonal-norm staggered SBP operators}

Instead of collocating the solution and integration nodes (which is what has been done up to this point), SBP operators can be constructed using two sets of nodes, as in the work of Parsani et al. [39] and the extension to multidimensional SBP operators in Ref. [19](in fact this idea can be generalized further to separate out the surface nodes; see the work of Chan [10,11] on modal decoupled SBP methods). The first is the solution nodes, $\tilde{\boldsymbol{\xi}}_{l}, l=1,2,3$ where the solution is stored and the second, $\boldsymbol{\xi}_{l}, l=1,2,3$ is where derivatives are computed. The idea is to construct SBP operators from an interpolation/extrapolation to the flux nodes, a computation of the derivative on the flux nodes, and an interpolation/extrapolation back to the solution nodes; the operators are referred to as staggered because the solution and flux nodes are staggered. For this purpose, the interpolation/extrapolation operators, $I_{S \operatorname{to} F, \xi_{l}}^{(1 D)}$ and $\mathbf{I}_{F \text { to } S, \xi_{l}}^{(1 D)}$ are introduced, which satisfy the following accuracy properties:

$\mathbf{I}_{S \mathrm{to} F, \xi_{l}}^{(1 D)} \tilde{\boldsymbol{\xi}}_{l}^{k}=\boldsymbol{\xi}_{l}^{k}, \quad \mathbf{I}_{F \mathrm{to}, \xi_{l}}^{(1 D)} \boldsymbol{\xi}_{l}^{j}=\tilde{\boldsymbol{\xi}}_{l}^{j}, k=0,1, \ldots, p, j=0,1, \ldots, p-1, l=1,2,3$.

Notice that the degree of $\mathbf{I}_{F \operatorname{to} S, \xi_{l}}^{(1 D)}$ is one less than that of $\mathbf{I}_{S \operatorname{to} F, \xi_{l}}^{(1 D)}$; this is because the differentiation operator on the flux nodes reduces the degree of the polynomial space that needs to be interpolated/extrapolated back to the solution nodes.

Furthermore, to obtain the SBP property, the interpolating/extrapolating operator $\mathbf{I}_{F \text { to } S, \xi_{l}}^{(1 D)}$ must satisfy [39]:

$$
\mathrm{I}_{F \mathrm{to} S, \xi_{l}}^{(1 D)}=\left(\tilde{\mathrm{H}}_{\xi_{l}}^{(1 D)}\right)^{-1}\left(\mathrm{I}_{S \operatorname{to} F, \xi_{l}}^{(1 D)}\right)^{\mathrm{T}} \mathrm{H}_{\xi_{l}}^{(1 D)} .
$$

A collocated SBP operator, $\tilde{\mathrm{D}}_{\xi_{l}}^{(1 D)}$, can be recast as

$$
\tilde{\mathrm{D}}_{\xi_{l}}^{(1 D)}=\mathbf{I}_{F \operatorname{to} S, \xi_{l}}^{(1 D)} \mathrm{D}_{\xi_{l}} \mathbf{I}_{S \operatorname{to} F, \xi_{l}}^{(1 D)}=\left(\tilde{\mathrm{H}}_{\xi_{l}}^{(1 D)}\right)^{-1}\left(\mathbf{I}_{S \operatorname{to} F, \xi_{l}}^{(1 D)}\right)^{\mathrm{T}} \mathbf{Q}_{\xi_{l}}^{(1 D)} \mathbf{I}_{S \operatorname{so} F, \xi_{l}}^{(1 D)}
$$

if a set of flux nodes can be found such that this is possible (see for example Theorem 7).

To see why the condition (26) is necessary to retain the SBP property, consider the decomposition (27) and define

$$
\tilde{\mathbf{Q}}_{\xi_{l}}^{(1 D)} \equiv \tilde{\mathrm{H}}_{\xi_{l}}^{(1 D)} \tilde{\mathrm{D}}_{\xi_{l}}^{(1 D)}=\left(\mathbf{I}_{S \operatorname{to} F, \xi_{l}}^{(1 D)}\right)^{\mathrm{T}} \mathbf{Q}_{\xi_{l}}^{(1 D)} \mathbf{I}_{S \operatorname{to} F, \xi_{l}}^{(1 D)}
$$


Furthermore, we have

$$
\tilde{\mathrm{Q}}_{\xi_{l}}^{(1 D)}+\tilde{\mathrm{Q}}_{\xi_{l}}^{(1 D)}=\left(\mathrm{I}_{S \operatorname{to} F, \xi_{l}}^{(1 D)}\right)^{\mathrm{T}}\left\{\mathrm{Q}_{\xi_{l}}^{(1 D)}+\left(\mathrm{Q}_{\xi_{l}}^{(1 D)}\right)^{\mathrm{T}}\right\} \mathrm{I}_{S \operatorname{to} F, \xi_{l}}^{(1 D)}=\left(\mathrm{I}_{S \operatorname{to} F, \xi_{l}}^{(1 D)}\right)^{\mathrm{T}} \mathrm{E}_{\xi_{l}}^{(1 D)} \mathbf{I}_{S \operatorname{to} F, \xi_{l}}^{(1 D)}
$$

and since the interpolation/extrapolation operator $\mathbf{I}_{S \operatorname{to} F, \xi_{l}}^{(1 D)}$ is at least degree $p$ accurate, $\tilde{\mathrm{E}}_{\xi_{l}}^{(1 D)} \equiv\left(\mathrm{I}_{S \operatorname{to} F, \xi_{l}}^{(1 D)}\right)^{\mathrm{T}} \mathrm{E}_{\xi_{l}}^{(1 D)} \mathrm{I}_{S \operatorname{to} F, \xi_{l}}^{(1 D)}$ satisfies the requirements of Definition 2 for an SBP operator.

A number of theorems are now presented regarding the existence of staggeredgrid SBP operators. The theorems here are a natural extension of the staggered grid work on multidimensional SBP operators of Ref. [19] to tensor-product operators. The following definition is first introduced

Definition 3 The degree $p$ Vandermonde matrix on set of nodes $\boldsymbol{\xi}_{l}$ is the matrix

$$
\mathrm{V}_{\xi_{l}, p}^{(1 D)}(:, k)=\boldsymbol{\xi}_{l}^{k}, \quad k=0,1, \ldots, p .
$$

The matrix of the derivatives of the monomials evaluated at mesh nodes associated with the Vandermonde matrix is given as

$$
\begin{aligned}
& \mathrm{V}_{\partial \xi_{1}, p}^{(1 D)}(:, k)=k \boldsymbol{\xi}_{1}^{k-1}, \quad \mathrm{~V}_{\partial \xi_{2}, p}^{(1 D)}(:, k)=k \boldsymbol{\xi}_{2}^{k-1}, \quad \mathrm{~V}_{\partial \xi_{3}, p}^{(1 D)}(:, k)=k \boldsymbol{\xi}_{3}^{k-1} \\
& k=0,1,2, \ldots, p
\end{aligned}
$$

If the collocated SBP/GSBP operator is of maximum degree, i.e., $p=N-1$, then it can always recast as a staggered grid SBP operator.

Theorem 7 All maximum degree (i.e. $p=N-1$ ) diagonal-norm GSBP operators can be decomposed as staggered-grid operators using any set of flux nodes on which there exists a quadrature rule with positive weights of at least degree $2 p-1$.

Proof On the flux nodes it is assumed that there exists a diagonal norm $\mathrm{H}_{\xi_{l}}^{(1 D)}$ that is at least degree $2 p-1$; this ensures that there exists at least a degree $p$ diagonal-norm SBP operator $\mathrm{D}_{\xi_{l}}^{(1 D)} \equiv\left(\mathrm{H}_{\xi_{l}}^{(1 D)}\right)^{-1} \mathrm{Q}_{\xi_{l}}^{(1 D)}[17]$.

The interpolation/extrapolation operators are constructed as

$$
\mathrm{I}_{S \operatorname{to} F, \xi_{l}}^{(1 D)}=\mathrm{V}_{\xi_{l}, p}^{(1 D)}\left(\tilde{\mathrm{V}}_{\xi_{l}, p}^{(1 D)}\right)^{-1}, \quad \mathrm{I}_{F \operatorname{to} S, \xi_{l}}^{(1 D)}=\left(\tilde{\mathrm{H}}_{\xi_{l}}^{(1 D)}\right)^{-1}\left(\mathrm{I}_{S \operatorname{to} F, \xi_{l}}^{(1 D)}\right)^{\mathrm{T}} \mathrm{H}_{\xi_{l}}^{(1 D)} \text {. }
$$

The interpolation/extrapolation operator, $\mathrm{I}_{S \operatorname{to} F, \xi_{l}}^{(1 D)}$, automatically satisfies the accuracy conditions (25), and it is therefore only necessary to demonstrate that $\mathbf{I}_{F \text { to } S, \xi_{l}}^{(1 D)}$ satisfies $(25)$ :

$$
\mathrm{I}_{F \mathrm{to} S, \xi_{l}}^{(1 D)} \boldsymbol{\xi}_{l}^{j}=\tilde{\boldsymbol{\xi}}_{l}^{j}, \quad j=1,2, \ldots, p-1 .
$$

Expanding $\mathbf{I}_{F \text { to } S, \xi_{l}}^{(1 D)}$ gives

$$
\left(\tilde{\mathbf{H}}_{\xi_{l}}^{(1 D)}\right)^{-1}\left\{\mathrm{~V}_{\xi_{l}, p}^{(1 D)}\left(\tilde{\mathbf{V}}_{\xi_{l}, p}^{(1 D)}\right)^{-1}\right\}^{\mathrm{T}} \mathrm{H}_{\xi_{l}}^{(1 D)} \boldsymbol{\xi}_{l}^{j}=\tilde{\boldsymbol{\xi}}_{l}^{j}
$$


multiplying both sides by $\left(\tilde{\mathrm{V}}_{\xi_{l}, p}^{(1 D)}\right)^{\mathrm{T}} \tilde{\mathrm{H}}_{\xi_{l}}^{(1 D)}$ leads to

$$
\left(\mathrm{V}_{\xi_{l}, p}^{(1 D)}\right)^{\mathrm{T}} \mathrm{H}_{\xi_{l}}^{(1 D)} \boldsymbol{\xi}_{l}^{j}=\left(\tilde{\mathrm{V}}_{\xi_{l}, p}^{(1 D)}\right)^{\mathrm{T}} \tilde{\mathrm{H}}_{\xi_{l}}^{(1 D)} \tilde{\boldsymbol{\xi}}_{l}^{j}
$$

where the final equality holds because both norm matrices are at least degree $2 p-1$ approximations to the $L^{2}$ inner product.

Finally, it is shown that $\tilde{\mathrm{D}}_{\xi_{l}}^{(1 D)}$ is recovered. Note that in the maximum degree case, the derivative operator is uniquely defined and given as $\tilde{\mathrm{D}}_{\xi_{l}}^{(1 D)}=$ $\tilde{\mathrm{V}}_{\partial \xi_{l}, p}^{(1 D)}\left(\tilde{\mathrm{V}}_{\xi_{l}, p}^{(1 D)}\right)^{-1}$

To show the equivalence, we start by applying $\mathbf{I}_{F \text { to } S, \xi_{l}}^{(1 D)} \mathrm{D}_{\xi_{l}}^{(1 D)} \mathbf{I}_{S \text { to } F, \xi_{l}}^{(1 D)}$ to the degree $p$ Vandermonde matrix This is equivalent to showing that the following equality holds:

$$
\begin{aligned}
\mathrm{I}_{F \mathrm{to} S, \xi_{l}}^{(1 D)} \mathrm{D}_{\xi_{l}}^{(1 D)} \mathrm{I}_{S \operatorname{to} F, \xi_{l}}^{(1 D)} \tilde{\mathrm{V}}_{\xi_{l}, p}^{(1 D)} & =\mathrm{I}_{F \operatorname{to} S, \xi_{l}}^{(1 D)} \mathrm{D}_{\xi_{l}}^{(1 D)} \mathrm{V}_{\xi_{l}, p}^{(1 D)} \\
= & \mathrm{I}_{F \operatorname{to} S, \xi_{l}}^{(1 D)} \mathrm{V}_{\partial \xi_{l}, p}^{(1 D)} \\
= & \tilde{\mathrm{V}}_{\partial \xi_{l}, p}^{(1 D)}
\end{aligned}
$$

where the polynomial exactness properties of $I_{S \text { to } F}$ and $I_{F \text { to } S}$ have been employed. Thus,

$$
\mathbf{I}_{F \operatorname{to} S, \xi_{l}}^{(1 D)} \mathrm{D}_{\xi_{l}}^{(1 D)} \mathbf{I}_{S \operatorname{to} F, \xi_{l}}^{(1 D)}=\tilde{\mathbf{V}}_{\partial \xi_{l}, p}^{(1 D)}\left(\tilde{\mathbf{V}}_{\xi_{l}, p}^{(1 D)}\right)^{-1}=\tilde{\mathrm{D}}_{\xi_{l}}^{(1 D)}
$$

In general, a set of flux nodes may or may not include boundary nodes, and the various permutations that have been considered can be employed in combination with staggering. To reduce the complexity, only the case where the flux node distribution includes boundary nodes is considered and therefore only one semi-discrete form is analyzed. If GSBP operators are used on the flux nodes, these are constructed using either the mortar element approach or the global SBP operator approach. Thus, with decomposition (27), the linear convection equation (6) is discretized as

$$
\begin{aligned}
& \operatorname{diag}(\tilde{\mathrm{J}})_{\kappa} \frac{\mathrm{d} \tilde{\boldsymbol{u}}_{\kappa}}{\mathrm{d} t} \\
& +\frac{1}{2} \tilde{\mathrm{H}}^{-1} \mathrm{I}_{S \operatorname{to} F}^{\mathrm{T}} \sum_{l, m=1}^{3}\left(\mathrm{Q}_{\xi_{l}} \operatorname{diag}\left(\mathcal{J} \frac{\partial \xi_{l}}{\partial x_{m}}\right)_{\kappa}+\operatorname{diag}\left(\mathcal{J} \frac{\partial \xi_{l}}{\partial x_{m}}\right)_{\kappa} \mathrm{Q}_{\xi_{l}}\right)_{\mathrm{I}_{S \operatorname{to} F} \tilde{\boldsymbol{u}}_{\kappa}=} \\
& \frac{1}{2} \tilde{\mathrm{H}}^{-1} \mathrm{I}_{S \operatorname{to} F}^{\mathrm{T}} \sum_{l, m=1}^{3}\left(\mathrm{E}_{\xi_{l}} \operatorname{diag}\left(\mathcal{J} \frac{\partial \xi_{l}}{\partial x_{m}}\right)_{\kappa} \mathrm{I}_{S \operatorname{to} F} \tilde{\boldsymbol{u}}_{\kappa}\right. \\
& +\operatorname{diag}\left(\mathcal{J} \frac{\partial \xi_{l}}{\partial x_{m}}\right)_{\kappa} \mathrm{R}_{\alpha_{\xi_{l}}^{\mathrm{T}}}^{\mathrm{T}} \mathrm{H}_{\xi_{l}}^{\perp} \mathrm{R}_{\beta_{\xi_{l}}} \mathrm{I}_{S \operatorname{to} F} \tilde{\boldsymbol{u}}_{(2 l-1)} \\
& \left.-\operatorname{diag}\left(\mathcal{J} \frac{\partial \xi_{l}}{\partial x_{m}}\right)_{\kappa} \mathrm{R}_{\beta_{\xi_{l}}^{\mathrm{T}}}^{\mathrm{T}} \mathrm{H}_{\xi_{l}}^{\perp} \mathrm{R}_{\alpha_{\xi_{l}}} \mathrm{I}_{S \mathrm{to} F} \tilde{\boldsymbol{u}}_{2 l}\right),
\end{aligned}
$$


where $\mathbf{I}_{S \text { to } F} \equiv \mathbf{I}_{S \text { to } F, \xi_{1}} \otimes \mathbf{I}_{S \text { to } F, \xi_{2}} \otimes \mathbf{I}_{S \text { to } F, \xi_{3}}$ and the metric terms are computed in the standard way on the flux nodes, for example the approaches in Refs. [22, $23,47,48]$. Moreover, $\tilde{\boldsymbol{u}}_{\kappa}$ is the vector containing the numerical solution at the solution nodes and similarly $\operatorname{diag}(\tilde{\mathrm{J}})$ is a diagonal matrix containing the metric Jacobian at the solution nodes. The following theorems summarize the stability and element-wise conservation of the staggered scheme:

Theorem 8 The semi-discrete scheme (28) is stable for the periodic problem and therefore for the nonperiodic problem with appropriate SATs for the weak imposition of boundary conditions.

Proof For the steps see Ref. [19].

Theorem 9 If the metric terms satisfy the following discrete form of the metric invariants (5):

$$
\sum_{l=1}^{3} \mathrm{D}_{\xi_{l}} \operatorname{diag}\left(\mathcal{J} \frac{\partial \xi_{l}}{\partial x_{m}}\right)_{\kappa} \mathbf{1}=\mathbf{0}, \quad m=1,2,3,
$$

then the semi-discrete scheme (28) is freestream preserving, element-wise conservative, and can be algebraically manipulated into the general telescoping flux form (11), where

$\bar{u}_{\kappa} \equiv \tilde{\mathbf{1}}^{\mathrm{T}} \tilde{\mathrm{H}} \operatorname{diag}(\tilde{\mathrm{J}})_{\kappa} \boldsymbol{u}_{\kappa}$,

$g_{\kappa}^{(2 l)} \equiv \frac{1}{2} \mathbf{1}^{\mathrm{T}} \sum_{l, m=1}^{3} \operatorname{diag}\left(\mathcal{J} \frac{\partial \xi_{l}}{\partial x_{m}}\right)_{\kappa}\left(\mathrm{R}_{\beta_{\xi_{l}}}^{\mathrm{T}} \mathrm{H}_{\xi_{l}}^{\perp} \mathrm{R}_{\beta_{\xi_{l}}} \boldsymbol{u}_{\kappa}+\mathrm{R}_{\beta_{\xi_{l}}}^{\mathrm{T}} \mathrm{H}_{\xi_{l}}^{\perp} \mathrm{R}_{\alpha_{\xi_{l}}} \boldsymbol{u}_{2 l}\right)$.

$g_{\kappa}^{(2 l-1)} \equiv-\frac{1}{2} \mathbf{1}^{\mathrm{T}} \sum_{l, m=1}^{3} \operatorname{diag}\left(\mathcal{J} \frac{\partial \xi_{l}}{\partial x_{m}}\right)_{\kappa}\left(\mathrm{R}_{\alpha_{\xi_{l}}}^{\mathrm{T}} \mathrm{H}_{\xi_{l}}^{\perp} \mathrm{R}_{\alpha_{\xi_{l}}} \boldsymbol{u}_{\kappa}+\mathrm{R}_{\alpha_{\xi_{l}}}^{\mathrm{T}} \mathrm{H}_{\xi_{l}}^{\perp} \mathrm{R}_{\beta_{\xi_{l}}} \boldsymbol{u}_{(2 l-1)}\right)$.

Proof For the steps see Ref. [19].

Remark 5 The discrete metric invariant conditions can be satisfied by using the approach given in Section 8.2 locally on each element.

\section{Stable discretizations with dense-norm SBP operators in curvilinear coordinates}

One of the drawbacks of diagonal-norm SBP operators is that finding $2 p-1$ accurate quadrature rules can in general be difficult and represents a restriction on the potential class of SBP operators that can be used. In contrast, dense-norm SBP operators have no such restriction; they always exist on a given set of nodes [17], and can be more accurate than mass-lumped operators [1]. Nevertheless, much of the work on SBP methods has been in the context of diagonal-norm operators because it was not clear how to construct linearly stable discretizations with dense-norm operators in curvilinear coordinates [45]. 
In this section, a theory for dense-norm tensor-product SBP operators is developed, and special SBP operators that incorporate the grid metric information directly are constructed. To do so, two sets of nodes are introduced. The solution is stored on the first set of nodes, defined by the vectors $\tilde{\boldsymbol{\xi}}_{l}, l=1,2,3$. The metrics are evaluated on the second set of nodes, which is defined by the vectors $\boldsymbol{\xi}_{l}, l=1,2,3$.

The first theorem gives a direct construction of dense-norm SBP operators from diagonal-norm SBP operators such that linearly stable schemes in curvilinear coordinates can be constructed.

Theorem 10 Consider two sets of nodes the solution nodes $\tilde{\boldsymbol{\xi}}_{l}$ and the flux nodes $\boldsymbol{\xi}_{l}, l=1,2,3$, of size $\tilde{n}$ and $n$, respectively, where $n \geq \tilde{n}$. If there exist degree $p$ diagonal-norm SBP operators on the flux nodes, $\mathrm{D}_{\xi_{l}}, l=1,2,3$, then order $p$ dense-norm tensor-product SBP operators can be constructed as

$\tilde{\mathrm{H}}_{\operatorname{diag}(\mathcal{J})_{\kappa}} \equiv \tilde{\mathrm{V}}_{(\tilde{n}-1)}^{-\mathrm{T}} \mathrm{V}_{(\tilde{n}-1)}^{\mathrm{T}} \mathrm{H} \operatorname{diag}(\mathcal{J})_{\kappa} \mathrm{V}_{(\tilde{n}-1)} \tilde{\mathrm{V}}_{(\tilde{n}-1)}^{-1}$,

$\tilde{\mathrm{Q}}_{\xi_{l}, m, \kappa} \equiv$

$\tilde{\mathrm{V}}_{(\tilde{n}-1)}^{-\mathrm{T}} \mathrm{V}_{(\tilde{n}-1)}^{\mathrm{T}}\left(\frac{1}{2} \mathrm{Q}_{\xi_{l}} \operatorname{diag}\left(\mathcal{J} \frac{\partial \xi_{l}}{\partial x_{m}}\right)_{\kappa}+\frac{1}{2} \operatorname{diag}\left(\mathcal{J} \frac{\partial \xi_{l}}{\partial x_{m}}\right)_{\kappa} \mathrm{Q}_{\xi_{l}}\right) \mathrm{V}_{(\tilde{n}-1)} \tilde{\mathrm{V}}_{(\tilde{n}-1)}^{-1}$,

$\tilde{\mathrm{E}}_{\xi_{l}, m, \kappa} \equiv$

$\tilde{\mathrm{V}}_{(\tilde{n}-1)}^{-\mathrm{T}} \mathrm{V}_{(\tilde{n}-1)}\left(\frac{1}{2} \mathrm{E}_{\xi_{l}} \operatorname{diag}\left(\mathcal{J} \frac{\partial \xi_{l}}{\partial x_{m}}\right)_{\kappa}+\frac{1}{2} \operatorname{diag}\left(\mathcal{J} \frac{\partial \xi_{l}}{\partial x_{m}}\right)_{\kappa} \mathrm{E}_{\xi_{l}}\right) \mathrm{V}_{(\tilde{n}-1)} \tilde{\mathrm{V}}_{(\tilde{n}-1)}^{-1}$

where

$$
\begin{aligned}
\tilde{\mathrm{V}}_{(\tilde{n}-1)} & =\tilde{\mathrm{V}}_{\xi_{1},(\tilde{n}-1)}^{(1 D)} \otimes \tilde{\mathrm{V}}_{\xi_{2},(\tilde{n}-1)}^{(1 D)} \otimes \tilde{\mathrm{V}}_{\xi_{3},(\tilde{n}-1)}^{(1 D)} \\
\mathrm{V}_{(\tilde{n}-1)} & =\mathrm{V}_{\xi_{1},(\tilde{n}-1)}^{(1 D)} \otimes \mathrm{V}_{\xi_{2},(\tilde{n}-1)}^{(1 D)} \otimes \mathrm{V}_{\xi_{3},(\tilde{n}-1)}^{(1 D)}
\end{aligned}
$$

Proof The first task is to show that the resulting derivative operator, $\tilde{\mathrm{D}}_{\xi_{l}, m, \kappa} \equiv$ $\tilde{\mathrm{H}}_{\operatorname{diag}(\mathcal{J})_{\kappa}}^{-1} \tilde{\mathbf{Q}}_{\xi_{l}, m, \kappa}$, is an order $p$ approximation to

$$
\frac{\mathcal{J}^{-1}}{2} \frac{\partial}{\partial \xi_{l}}\left(\mathcal{J} \frac{\partial \xi_{l}}{\partial x_{m}} \mathcal{U}\right)+\frac{1}{2} \frac{\partial \xi_{l}}{\partial x_{m}} \frac{\partial \mathcal{U}}{\partial \xi_{l}}
$$


To do so, $\tilde{\mathrm{D}}_{\xi_{l}, m, \kappa}$ is applied to the degree $p$ Vandermonde matrix, $\tilde{\mathrm{V}}_{p}$, on the $\tilde{\boldsymbol{\xi}}_{l}, l=1,2,3$ nodes, which gives

$$
\begin{gathered}
\tilde{\mathrm{D}}_{\xi_{l}, m, \kappa} \tilde{\mathrm{V}}_{p}=\tilde{\mathrm{V}}_{(\tilde{n}-1)}\left(\mathrm{V}_{(\tilde{n}-1)}^{\mathrm{T}} \mathrm{H} \operatorname{diag}(\mathcal{J})_{\kappa} \mathrm{V}_{(\tilde{n}-1)}\right)^{-1} \tilde{\mathrm{V}}_{(\tilde{n}-1)}^{\mathrm{T}} \mathrm{H} \operatorname{diag}(\mathcal{J})_{\kappa} \\
\left(\frac{1}{2} \operatorname{diag}(\mathcal{J})_{\kappa}^{-1} \mathrm{D}_{\xi_{l}} \operatorname{diag}\left(\mathcal{J} \frac{\partial \xi_{l}}{\partial x_{m}}\right)_{\kappa}+\frac{1}{2} \operatorname{diag}(\mathcal{J})_{\kappa}^{-1} \operatorname{diag}\left(\mathcal{J} \frac{\partial \xi_{l}}{\partial x_{m}}\right)_{\kappa} \mathrm{D}_{\xi_{l}}\right) \mathrm{V}_{p}
\end{gathered}
$$

where $\mathrm{V}_{=} \mathrm{V}_{\xi_{1}, p}^{(1 D)} \otimes \mathrm{V}_{\xi_{2}, p}^{(1 D)} \otimes \mathrm{V}_{\xi_{3}, p}^{(1 D)}$. The matrix

$$
\tilde{\mathrm{V}}_{(\tilde{n}-1)}\left(\mathrm{V}_{(\tilde{n}-1)}^{\mathrm{T}} \mathrm{H} \operatorname{diag}(\mathcal{J})_{\kappa} \mathrm{V}_{(\tilde{n}-1)}\right)^{-1} \tilde{\mathrm{V}}_{(\tilde{n}-1)} \mathrm{H} \operatorname{diag}(\mathcal{J})_{\kappa}
$$

is at least a degree $p$ projection operator from the flux nodes to the solution nodes, i.e.,

$$
\tilde{\mathrm{V}}_{(\tilde{n}-1)}\left(\mathrm{V}_{(\tilde{n}-1)}^{\mathrm{T}} \mathrm{H} \operatorname{diag}(\mathcal{J})_{\kappa} \mathrm{V}_{(\tilde{n}-1)}\right)^{-1} \tilde{\mathrm{V}}_{(\tilde{n}-1)} \mathrm{H} \operatorname{diag}(\mathcal{J})_{\kappa} \mathrm{V}_{p}=\tilde{\mathrm{V}}_{p}
$$

Thus, the action of

$$
\tilde{\mathrm{V}}_{(\tilde{n}-1)}\left(\mathrm{V}_{(\tilde{n}-1)}^{\mathrm{T}} \mathrm{H} \operatorname{diag}(\mathcal{J})_{\kappa} \mathrm{V}_{(\tilde{n}-1)}\right)^{-1} \tilde{\mathrm{V}}_{(\tilde{n}-1)} \mathrm{H} \operatorname{diag}(\mathcal{J})_{\kappa}
$$

on the right-hand side of $(30)$ is to project

$$
\left(\frac{1}{2} \operatorname{diag}(\mathcal{J})_{\kappa}^{-1} \mathrm{D}_{\xi_{l}} \operatorname{diag}\left(\mathcal{J} \frac{\partial \xi_{l}}{\partial x_{m}}\right)_{\kappa}+\frac{1}{2} \operatorname{diag}(\mathcal{J})_{\kappa}^{-1} \operatorname{diag}\left(\mathcal{J} \frac{\partial \xi_{l}}{\partial x_{m}}\right)_{\kappa} \mathrm{D}_{\xi_{l}}\right) \mathrm{V}_{p}
$$

onto the nodes $\tilde{\boldsymbol{\xi}}_{l}, l=1,2,3$, which shows that $\tilde{\mathrm{D}}_{\xi_{l}, m, \kappa}$ is an order $p$ approximation. What remains is to show that the proposed construction leads to an SBP property, which easily follows, as a result of using a skew-symmetric splitting on the flux nodes.

Remark 6 In Theorem 10, for simplicity, we have used the Vandermonde matrices $\tilde{\mathrm{V}}_{(\tilde{n}-1)}$ and $\mathrm{V}_{(\tilde{n}-1)}$. However, to introduce additional degrees of freedom and for an easy extension to multidimensional SBP operators, where an invertible Vandermonde matrix may not exist, the theorem extends to decomposing the norm matrix as

$$
\tilde{\mathrm{H}}_{\operatorname{diag}(\mathcal{J})_{\kappa}} \equiv \tilde{\mathrm{W}}^{-\mathrm{T}} \mathrm{W}^{\mathrm{T}} \mathrm{H} \operatorname{diag}(\mathcal{J})_{\kappa} \mathrm{W} \tilde{\mathrm{W}}, \quad \tilde{\mathrm{W}} \equiv\left[\tilde{\mathrm{V}}_{p}, \tilde{\mathrm{Y}}\right], \quad \mathrm{W} \equiv\left[\mathrm{V}_{p}, \mathrm{Y}\right]
$$

where $\tilde{Y}$ and $Y$ are constructed such that $\tilde{W}$ and $W$ have linearly independent columns (this can always be accomplished) and therefore $\tilde{W}$ is invertible. The remaining matrices of the SBP operator would be constructed in the same way as in the theorem, swapping out $\tilde{\mathrm{V}}_{(\tilde{n}-1)}$ and $\mathrm{V}_{(\tilde{n}-1)}$ with $\tilde{\mathrm{W}}$ and $\mathrm{W}$, respectively.

Finally, the following existence theorem is presented: 
1 Theorem 11 Given a set of solution nodes $\tilde{\boldsymbol{\xi}}_{l}, l=1,2,3$, of size $\tilde{n}$, then there exist degree $1 \leq p \leq \tilde{n}-1$ dense-norm SBP operators as given in Theorem 10 .

Proof The flux mesh can always be constructed, $\boldsymbol{\xi}_{l}, l=1,2,3$, so that appropriate $\mathrm{H}, \mathrm{Q}_{\xi_{l}}$ and $\mathrm{E}_{\xi_{l}}$ matrices can be constructed, for example using tensorproduct Gauss-Lobatto nodes.

\subsection{Relation to the staggered approach}

The class of dense-norm SBP operators considered in the previous section are those that can be decomposed by introducing a second mesh in an analogous fashion to the staggered approach. As it turns out, this decomposition can be viewed in the terminology of the staggered approach. Consider defining

$$
\mathrm{I}_{S \mathrm{to} F} \equiv \mathrm{V}_{(\tilde{n}-1)} \tilde{\mathrm{V}}_{(\tilde{n}-1)}^{-1}
$$

then

$$
\begin{aligned}
& \tilde{\mathrm{H}}_{\operatorname{diag}(\mathcal{J})_{\kappa}}=\mathrm{I}_{\text {Sto } F}^{\mathrm{T}} \mathrm{H} \operatorname{diag}(\mathcal{J})_{\kappa} \mathrm{I}_{S \text { to } F}, \\
& \tilde{\mathrm{Q}}_{\xi_{l}, m, \kappa}=\mathrm{I}_{\text {Sto } F}^{\mathrm{T}}\left(\frac{1}{2} \mathrm{Q}_{\xi_{l}} \operatorname{diag}\left(\mathcal{J} \frac{\partial \xi_{l}}{\partial x_{m}}\right)_{\kappa}+\frac{1}{2} \operatorname{diag}\left(\mathcal{J} \frac{\partial \xi_{l}}{\partial x_{m}}\right)_{\kappa} \mathrm{Q}_{\xi_{l}}\right) \mathrm{I}_{S \text { to } F}, \\
& \tilde{\mathrm{E}}_{\xi_{l}, m, \kappa}=\mathrm{I}_{S \operatorname{to} F}^{\mathrm{T}}\left(\frac{1}{2} \mathrm{E}_{\xi_{l}} \operatorname{diag}\left(\mathcal{J} \frac{\partial \xi_{l}}{\partial x_{m}}\right)_{\kappa}+\frac{1}{2} \operatorname{diag}\left(\mathcal{J} \frac{\partial \xi_{l}}{\partial x_{m}}\right)_{\kappa} \mathrm{E}_{\xi_{l}}\right) \mathrm{I}_{S \text { to } F} .
\end{aligned}
$$

The SBP requirements on $\mathrm{I}_{F \text { to } S}(26)$ result in

$$
\mathrm{I}_{F \operatorname{to} S}=\tilde{\mathrm{H}}_{\mathcal{J}_{\kappa}}^{-1} \mathrm{I}_{S \operatorname{to} F}^{\mathrm{T}} \mathrm{H} \operatorname{diag}(\mathcal{J})_{\kappa} \cdot
$$

It is necessary to show that $\mathbf{I}_{F \text { to } S}$ is at least a degree $p-1$ projection from the flux nodes to the solution nodes. First applying $I_{F \text { to } S}$ to the degree $p$ Vandermonde matrix on the flux nodes gives

$$
\begin{aligned}
\mathrm{I}_{F \operatorname{to} S} \mathrm{~V}_{p} & =\tilde{\mathrm{H}}_{\mathcal{J}_{\kappa}}^{-1} \mathrm{I}_{S \operatorname{to} F}^{\mathrm{T}} \mathrm{H} \operatorname{diag}(\mathcal{J})_{\kappa} \mathrm{V}_{p}, \\
& =\tilde{\mathrm{V}}_{\tilde{n}}\left(\mathrm{~V}_{\tilde{n}}^{\mathrm{T}} \mathrm{H} \operatorname{diag}(\mathcal{J})_{\kappa} \mathrm{V}_{\tilde{n}}\right)^{-1} \tilde{\mathrm{V}}_{\tilde{n}}^{\mathrm{T}} \tilde{\mathrm{V}}_{\tilde{n}}^{-\mathrm{T}} \mathrm{V}_{\tilde{n}}^{\mathrm{T}} \mathrm{H} \operatorname{diag}(\mathcal{J})_{\kappa} \mathrm{V}_{p} \\
& =\tilde{\mathrm{V}}_{\tilde{n}}\left(\mathrm{~V}_{\tilde{n}}^{\mathrm{T}} \mathrm{H} \operatorname{diag}(\mathcal{J})_{\kappa} \mathrm{V}_{\tilde{n}}\right)^{-1} \mathrm{~V}_{\tilde{n}}^{\mathrm{T}} \mathrm{H} \operatorname{diag}(\mathcal{J})_{\kappa} \mathrm{V}_{p} \\
& =\tilde{\mathrm{V}}_{p},
\end{aligned}
$$

Thus, $\mathrm{I}_{F \text { to } S}$ is of degree $p$. 
Finally,

$$
\begin{aligned}
& \tilde{\mathrm{D}}_{\xi_{l}, m, \kappa}=\tilde{\mathrm{H}}_{\mathcal{J}_{\kappa}}^{-1} \tilde{Q}_{\xi_{l}, m, \kappa}, \\
& =\left.\tilde{\mathrm{H}}_{\mathcal{J}_{\kappa}}^{-1}\right|_{S \operatorname{Sto} F} ^{\mathrm{T}}\left(\frac{1}{2} Q_{\xi_{l}} \operatorname{diag}\left(\mathcal{J} \frac{\partial \xi_{l}}{\partial x_{m}}\right)_{\kappa}+\frac{1}{2} \operatorname{diag}\left(\mathcal{J} \frac{\partial \xi_{l}}{\partial x_{m}}\right)_{\kappa} \mathrm{Q}_{\xi_{l}}\right) \mathrm{I}_{S \mathrm{to} F}, \\
& =\left.\tilde{\mathrm{H}}_{\mathcal{J}_{\kappa}}^{-1}\right|_{S \operatorname{to} F} ^{\mathrm{T}} \mathrm{H} \operatorname{diag}(\mathcal{J})_{\kappa}\left(\frac{1}{2} \operatorname{diag}(\mathcal{J})_{\kappa}^{-1} \mathrm{D}_{\xi_{l}} \operatorname{diag}\left(\mathcal{J} \frac{\partial \xi_{l}}{\partial x_{m}}\right)_{\kappa}\right. \\
& \left.+\frac{1}{2} \operatorname{diag}(\mathcal{J})_{\kappa}^{-1} \operatorname{diag}\left(\mathcal{J} \frac{\partial \xi_{l}}{\partial x_{m}}\right)_{\kappa} \mathrm{D}_{\xi_{l}}\right) \mathrm{I}_{S \operatorname{to} F}, \\
& =\mathrm{I}_{F \operatorname{to} S}\left(\frac{1}{2} \operatorname{diag}(\mathcal{J})_{\kappa}^{-1} \mathrm{D}_{\xi_{l}} \operatorname{diag}\left(\mathcal{J} \frac{\partial \xi_{l}}{\partial x_{m}}\right)_{\kappa}\right. \\
& \left.+\frac{1}{2} \operatorname{diag}(\mathcal{J})_{\kappa}^{-1} \operatorname{diag}\left(\mathcal{J} \frac{\partial \xi_{l}}{\partial x_{m}}\right)_{\kappa} \mathrm{D}_{\xi_{l}}\right) \mathrm{I}_{S \operatorname{to} F},
\end{aligned}
$$

and it is clear that the two approaches are very similar, except for the contribution of the metric Jacobian. When viewing the semi-discrete form as an approximation to the weak form, the dense-norm approach is an over-integration on both the temporal and volume terms, while the staggered approach using diagonal-norm SBP operators is only an over-integration on the volume terms.

Remark 7 We note the connection between our construction of $\tilde{\mathrm{H}}_{\mathcal{J}_{\kappa}}^{-1}$ and the work of Ranocha et al. [43]: On the solution nodes, we construct, from $\tilde{\mathrm{H}}_{\mathcal{J}_{\kappa}}^{-1}$, the dense matrix $\tilde{J}$ as

$$
\tilde{\mathrm{J}} \equiv \tilde{\mathrm{H}}^{-1} \tilde{\mathbf{H}}_{\mathcal{J}_{\kappa}}=\mathrm{I}_{F \operatorname{toS}} \operatorname{diag}(\mathcal{J})_{\kappa} \mathrm{l}_{S \mathrm{to} F},
$$

which satisfies the conditions required by Ranocha et al. [43], i.e.,

$$
\tilde{\mathrm{H}} \tilde{J}=(\tilde{H} \tilde{J})^{\mathrm{T}}, \quad \tilde{\mathrm{H}} \tilde{J}>0 .
$$

Thus, if we consider a nodal basis based SBP operator on the flux nodes and a nodal basis on the solution nodes (both having $n=p+1$ ), then the resultant $\tilde{J}$ is identical to that of Ranocha et al. [43].

\section{Interface dissipation and SATs for boundary conditions}

Thus far, the SATs that have been considered lead to neutrally-stable schemes. In this section, these SATs are augmented by interface dissipation (for linear problems this is important because upwinding can result in $p+1$ convergence rates; in addition, for nonlinear problems upwinding can provide increased robustness). The discussion will focus on diagonal-norm GSBP operators and constructing the dissipative terms on the mortar. These ideas can then be straightforwardly applied to the remaining approaches covered in the paper. The goal is to construct dissipative terms that result in upwind SATs that 
augment the neutral stability of the symmetric SAT and that locally can be viewed as weakly imposed well-posed boundary conditions.

The upwind SATs in flux form are constructed as

$$
\boldsymbol{S A T} \boldsymbol{s} \equiv \mathrm{H}^{-1} \sum_{l=1}^{3}\left\{-\mathrm{R}_{\alpha_{\xi_{l}}}^{\mathrm{T}} \mathrm{H}_{\xi_{l}}^{\perp}\left(\boldsymbol{f}_{(2 l-1)}-\boldsymbol{f}_{(2 l-1)}^{I}\right)+\mathrm{R}_{\beta_{\xi_{l}}}^{\mathrm{T}} \mathrm{H}_{\xi_{l}}^{\perp}\left(\boldsymbol{f}_{2 l}-\boldsymbol{f}_{2 l}^{I}\right)\right\},
$$

where the fluxes are given as

$$
\boldsymbol{f}_{(2 l-1)} \equiv \sum_{m=1}^{3} \mathrm{R}_{\alpha_{\xi_{l}}} \operatorname{diag}\left(\mathcal{J} \frac{\partial \xi_{l}}{\partial x_{m}}\right)_{\kappa} \boldsymbol{u}_{\kappa}, \quad \boldsymbol{f}_{2 l} \equiv \sum_{m=1}^{3} \mathrm{R}_{\beta_{\xi_{l}}} \operatorname{diag}\left(\mathcal{J} \frac{\partial \xi_{l}}{\partial x_{m}}\right)_{\kappa} \boldsymbol{u}_{\kappa},
$$

and the numerical fluxes are given as

$$
\begin{aligned}
\boldsymbol{f}_{(2 l-1)}^{I} \equiv & \frac{1}{2}\left(\Lambda_{(2 l-1)}^{\kappa} \mathrm{R}_{\beta_{\xi_{l}}} \boldsymbol{u}_{(2 l-1)}+\mathrm{R}_{\alpha_{\xi_{l}}} \sum_{m=1}^{3} \operatorname{diag}\left(\mathcal{J} \frac{\partial \xi_{l}}{\partial x_{m}}\right)_{\kappa} \boldsymbol{u}_{\kappa}\right) \\
& -\frac{1}{2}\left|\Lambda_{(2 l-1)}^{\kappa}\right|\left(\mathrm{R}_{\alpha_{\xi_{l}}} \boldsymbol{u}_{\kappa}-\mathrm{R}_{\beta_{\xi_{l}}} \boldsymbol{u}_{(2 l-1)}\right) \\
\boldsymbol{f}_{2 l}^{I} \equiv & \frac{1}{2}\left(\mathrm{R}_{\beta_{\xi_{l}}} \sum_{m=1}^{3} \operatorname{diag}\left(\mathcal{J} \frac{\partial \xi_{l}}{\partial x_{m}}\right)_{\kappa} \boldsymbol{u}_{\kappa}+\Lambda_{2 l}^{\kappa} \mathrm{R}_{\alpha_{\xi_{l}}} \boldsymbol{u}_{2 l}\right)-\frac{1}{2}\left|\Lambda_{2 l}^{\kappa}\right|\left(\mathrm{R}_{\alpha_{\xi_{l}}} \boldsymbol{u}_{2 l}-\mathrm{R}_{\beta_{\xi_{l}}} \boldsymbol{u}_{\kappa}\right),
\end{aligned}
$$

where

$$
\begin{aligned}
& \Lambda_{(2 l-1)}^{\kappa} \equiv \sum_{m=1}^{3} \operatorname{diag}\left(\mathcal{J} \frac{\partial \xi_{l}}{\partial x_{m}}\right)^{(2 l-1)}, \quad \Lambda_{2 l}^{\kappa} \equiv \sum_{m=1}^{3} \operatorname{diag}\left(\mathcal{J} \frac{\partial \xi_{l}}{\partial x_{m}}\right)^{2 l} \\
& \left|\Lambda_{(2 l-1)}^{\kappa}\right| \equiv\left|\sum_{m=1}^{3} \operatorname{diag}\left(\mathcal{J} \frac{\partial \xi_{l}}{\partial x_{m}}\right)^{(2 l-1)}\right|, \quad\left|\Lambda_{2 l}^{\kappa}\right| \equiv\left|\sum_{m=1}^{3} \operatorname{diag}\left(\mathcal{J} \frac{\partial \xi_{l}}{\partial x_{m}}\right)^{2 l}\right| .
\end{aligned}
$$

Inserting (34) and (35) into (33) gives, after rearrangement:

$S A T s=$

$\frac{1}{2} \mathrm{H}^{-1} \sum_{l, m=1}^{3}\left(\mathrm{E}_{\xi_{l}} \operatorname{diag}\left(\mathcal{J} \frac{\partial \xi_{l}}{\partial x_{m}}\right)_{\kappa} \boldsymbol{u}_{\kappa}\right)$

$+\frac{1}{2} \mathrm{H}^{-1} \sum_{l, m=1}^{3}\left(\mathrm{R}_{\alpha_{\xi_{l}}}^{\mathrm{T}} \mathrm{H}_{\xi_{l}}^{\perp} \operatorname{diag}\left(\mathcal{J} \frac{\partial \xi_{l}}{\partial x_{m}}\right)^{(2 l-1)} \mathrm{R}_{\beta_{\xi_{l}}} \boldsymbol{u}_{(2 l-1)}-\mathrm{R}_{\beta_{\xi_{l}}}^{\mathrm{T}} \mathrm{H}_{\xi_{l}}^{\perp} \operatorname{diag}\left(\mathcal{J} \frac{\partial \xi_{l}}{\partial x_{m}}\right)^{2 l} \mathrm{R}_{\alpha_{\xi_{l}}} \boldsymbol{u}_{2 l}\right)$

$+\frac{1}{2} \sum_{l=1}^{3}\left\{-\mathrm{R}_{\alpha_{\xi_{l}}}^{\mathrm{T}} \mathrm{H}_{\xi_{l}}^{\perp}\left|\Lambda_{(2 l-1)}^{\kappa}\right|\left(\mathrm{R}_{\alpha_{\xi_{l}}} \boldsymbol{u}_{\kappa}-\mathrm{R}_{\beta_{\xi_{l}}} \boldsymbol{u}_{(2 l-1)}\right)+\mathrm{R}_{\beta_{\xi_{l}}}^{\mathrm{T}} \mathrm{H}_{\xi_{l}}^{\perp}\left|\Lambda_{2 l}^{\kappa}\right|\left(\mathrm{R}_{\alpha_{\xi_{l}}} \boldsymbol{u}_{2 l}-\mathrm{R}_{\beta_{\xi_{l}}} \boldsymbol{u}_{\kappa}\right)\right\}$

where the identity $\mathrm{E}_{\xi_{l}}=\sum_{l=1}^{3}\left(-\mathrm{R}_{\alpha_{\xi_{l}}}^{\mathrm{T}} \mathrm{H}_{\xi_{l}}^{\perp} \mathrm{R}_{\alpha_{\xi_{l}}}+\mathrm{R}_{\beta_{\xi_{l}}^{\mathrm{T}}}^{\mathrm{T}} \mathrm{H}_{\xi_{l}}^{\perp} \mathrm{R}_{\beta_{\xi_{l}}}\right)$ has been used.

Thus, the form of the dissipation is 


$$
\boldsymbol{d i s} \equiv \frac{1}{2} \mathrm{H}^{-1} \sum_{l=1}^{3}\left\{-\mathrm{R}_{\alpha_{\xi_{l}}}^{\mathrm{T}} \mathrm{H}_{\xi_{l}}^{\perp}\left|\Lambda_{(2 l-1)}^{\kappa}\right|\left(\mathrm{R}_{\alpha_{\xi_{l}}} \boldsymbol{u}_{\kappa}-\mathrm{R}_{\beta_{\xi_{l}}} \boldsymbol{u}_{(2 l-1)}\right)+\mathrm{R}_{\beta_{\xi_{l}}}^{\mathrm{T}} \mathrm{H}_{\xi_{l}}^{\perp}\left|\Lambda_{2 l}^{\kappa}\right|\left(\mathrm{R}_{\alpha_{\xi_{l}}} \boldsymbol{u}_{2 l}-\mathrm{R}_{\beta_{\xi_{l}}} \boldsymbol{u}_{\kappa}\right)\right\}
$$

The next theorem states that the dissipation has the desired properties.

Theorem 12 The dissipation terms (36) are design order and retain the energy stability and element-wise conservation properties of the base schemes.

Proof Design order follows by inserting the projections of polynomials onto the mesh into (36). To prove that the additional terms maintain stability, consider the terms associated with the $2 l$ surface. At this surface, the contribution from the two abutting elements from the energy analysis is

$\boldsymbol{u}_{\kappa}^{\mathrm{T}} \mathrm{H} \boldsymbol{d i s s _ { \kappa }}+\boldsymbol{u}_{2 l} \mathrm{H}^{\mathrm{T}} \boldsymbol{d i s s}_{2 l}=$

$\frac{1}{2} \sum_{m=1}^{3}\left\{\boldsymbol{u}_{\kappa}^{\mathrm{T}} \mathrm{R}_{\alpha_{\xi_{l}}}^{\mathrm{T}} \mathrm{H}_{\xi_{l}}^{\perp}\left|\Lambda_{2 l}^{\kappa}\right|\left(\mathrm{R}_{\alpha_{\xi_{l}}} \boldsymbol{u}_{2 l}-\mathrm{R}_{\beta_{\xi_{l}}} \boldsymbol{u}_{\kappa}\right)-\boldsymbol{u}_{2 l}^{\mathrm{T}} \mathrm{R}_{\alpha_{\xi_{l}}}^{\mathrm{T}} \mathrm{H}_{\xi_{l}}^{\perp}\left|\Lambda_{(2 l-1)}^{\kappa}\right|\left(\mathrm{R}_{\alpha_{\xi_{l}}} \boldsymbol{u}_{2 l}-\mathrm{R}_{\beta_{\xi_{l}}} \boldsymbol{u}_{\kappa}\right)\right\}$.

Defining $\tilde{\boldsymbol{u}}_{\kappa} \equiv \mathrm{R}_{\beta_{\xi_{l}}}^{\mathrm{T}} \boldsymbol{u}_{\kappa}, \tilde{\boldsymbol{u}}_{2 l} \equiv \mathrm{R}_{\alpha_{\xi_{l}}}^{\mathrm{T}} \boldsymbol{u}_{2 l}$, and $\mathrm{M} \equiv \sum_{l=1}^{3} \mathrm{H}_{\xi_{l}}^{\perp} \operatorname{diag}\left(\left|\mathcal{J} \frac{\partial \xi_{l}}{\partial x_{m}}\right|\right)^{2 l}$, the above reduces to

$$
\boldsymbol{u}_{\kappa}^{\mathrm{T}} \mathrm{H} \boldsymbol{d i s s _ { \kappa }}+\boldsymbol{u}_{2 l}^{\mathrm{T}} \mathrm{H} \boldsymbol{d i s s} \boldsymbol{s}_{2 l}=\frac{1}{2}\left[\begin{array}{ll}
\tilde{\boldsymbol{u}}_{\kappa}^{\mathrm{T}} & \tilde{\boldsymbol{u}}_{2 l}^{\mathrm{T}}
\end{array}\right] \mathrm{M} \otimes\left[\begin{array}{cc}
-1 & 1 \\
1 & -1
\end{array}\right]\left[\begin{array}{c}
\tilde{\boldsymbol{u}}_{\kappa} \\
\tilde{\boldsymbol{u}}_{2 l}
\end{array}\right] \leq 0 .
$$

To prove element-wise conservation it is necessary to demonstrate that the dissipation terms do not destroy the telescoping property of the base scheme. Again, concentrating on the $2 l$ surface, the contributions from the two abutting elements to the telescoping analysis are

$\mathbf{1}^{\mathrm{T}} \mathrm{Hdiss}_{\kappa}+\mathbf{1}^{\mathrm{T}} \mathrm{Hdiss}_{2 l}=$ $\frac{1}{2} \sum_{m=1}^{3}\left\{\mathbf{1}^{\mathrm{T}} \mathrm{R}_{\alpha_{\xi_{l}}}^{\mathrm{T}} \mathrm{H}_{\xi_{l}}^{\perp}\left|\Lambda_{2 l}^{\kappa}\right|\left(\mathrm{R}_{\alpha_{\xi_{l}}} \boldsymbol{u}_{2 l}-\mathrm{R}_{\beta_{\xi_{l}}} \boldsymbol{u}_{\kappa}\right)-\mathbf{1}^{\mathrm{T}} \mathrm{R}_{\alpha_{\xi_{l}}}^{\mathrm{T}} \mathrm{H}_{\xi_{l}}^{\perp}\left|\Lambda_{(2 l-1)}^{\kappa}\right|\left(\mathrm{R}_{\alpha_{\xi_{l}}} \boldsymbol{u}_{2 l}-\mathrm{R}_{\beta_{\xi_{l}}} \boldsymbol{u}_{\kappa}\right)\right\}=0$,

where $\mathbf{1}^{\mathrm{T}} \mathrm{R}_{\beta_{\xi_{l}}}^{\mathrm{T}}=\mathbf{1}^{\mathrm{T}} \mathrm{R}_{\alpha_{\xi_{l}}}^{\mathrm{T}}$ has been used. Consistency follows from the fact that the dissipation terms are design order and boundedness follows readily (see Ref. [18]).

The SATs constructed to impose upwinding are based on locally approximating well-posed boundary conditions. Therefore, their structure can be reused to impose characteristic boundary conditions, where the abutting element's solution is replaced with a known state. Thus, for discretization (15), 
the SAT on the $2 l-1$ face takes the form

$\boldsymbol{S} \boldsymbol{A} \boldsymbol{T}_{(2 l-1)}=$

$-\frac{1}{2} \mathrm{H}^{-1} \sum_{m=1}^{3}\left(\mathrm{R}_{\alpha_{\xi_{l}}}^{\mathrm{T}} \mathrm{H}_{\xi_{l}}^{\perp} \mathrm{R}_{\alpha_{\xi_{l}}} \operatorname{diag}\left(\mathcal{J} \frac{\partial \xi_{l}}{\partial x_{m}}\right)_{\kappa} \boldsymbol{u}_{\kappa}-\mathrm{R}_{\alpha_{\xi_{l}}}^{\mathrm{T}} \mathrm{H}_{\xi_{l}}^{\perp} \operatorname{diag}\left(\mathcal{J} \frac{\partial \xi_{l}}{\partial x_{m}}\right)^{(2 l-1)} \boldsymbol{g}\right)$

$-\frac{1}{2} \mathrm{H}^{-1} \mathrm{R}_{\alpha_{\xi_{l}}}^{\mathrm{T}} \mathrm{H}_{\xi_{l}}^{\perp}\left|\Lambda_{(2 l-1)}^{\kappa}\right|\left(\mathrm{R}_{\alpha_{\xi_{l}}} \boldsymbol{u}_{\kappa}-\mathrm{R}_{\beta_{\xi_{l}}} \boldsymbol{g}\right)$,

while the SAT on the $2 l$ face takes the form

$\boldsymbol{S A T} \boldsymbol{T}_{2 l}=$

$\frac{1}{2} \mathrm{H}^{-1} \sum_{m=1}^{3}\left(\mathrm{R}_{\beta_{\xi_{l}}}^{\mathrm{T}} \mathrm{H}_{\xi_{l}}^{\perp} \mathrm{R}_{\beta_{\xi_{l}}} \operatorname{diag}\left(\mathcal{J} \frac{\partial \xi_{l}}{\partial x_{m}}\right)_{\kappa} \boldsymbol{u}_{\kappa}-\mathrm{R}_{\beta_{\xi_{l}}}^{\mathrm{T}} \mathrm{H}_{\xi_{l}}^{\perp} \operatorname{diag}\left(\mathcal{J} \frac{\partial \xi_{l}}{\partial x_{m}}\right)^{2 l} \boldsymbol{g}\right)$

$\frac{1}{2} \mathrm{H}^{-1} \mathrm{R}_{\beta_{\xi_{l}}^{\mathrm{T}}}^{\mathrm{T}} \mathrm{H}_{\xi_{l}}^{\perp}\left|\Lambda_{2 l}^{\kappa}\right|\left(\mathrm{R}_{\alpha_{\xi_{l}}} \boldsymbol{g}-\mathrm{R}_{\beta_{\xi_{l}}} \boldsymbol{u}_{\kappa}\right)$,

where $\boldsymbol{g}$ is a vector constructed from the known state $\mathcal{G}$ evaluated at the mortar nodes.

The final theorem in this section demonstrates that the boundary SATs lead to energy stability.

Theorem 13 The boundary SATs (37) and (38) result in an energy stable scheme.

Proof The proof follows standard energy analysis.

\section{Numerical examples}

Examples based on the three-dimensional linear convection equation (4) are presented in order to demonstrate the theory introduced above. The initial condition is

$$
\mathcal{U}_{0}(x, y, z)=\sin (2 \pi x)+\sin (2 \pi y)+\sin (2 \pi z)
$$

and boundary conditions are periodic on all faces. The computational domain is obtained using the transformation (See example in Figure 3):

$$
\begin{aligned}
& x=\xi+\frac{1}{5} \sin (\pi \xi) \sin (\pi \eta) \\
& y=\eta+\frac{1}{5} \exp (1-\eta) \sin (\pi \xi) \sin (\pi \eta) \\
& z=\zeta+\frac{1}{20}[\sin (2 \pi x)+\sin (2 \pi y)]
\end{aligned}
$$

where the underlying computational space is a unit cube $[\xi, \eta, \zeta] \in[0,1]^{3}$. This transformation is a modified version of the one used in [21]. The distribution 

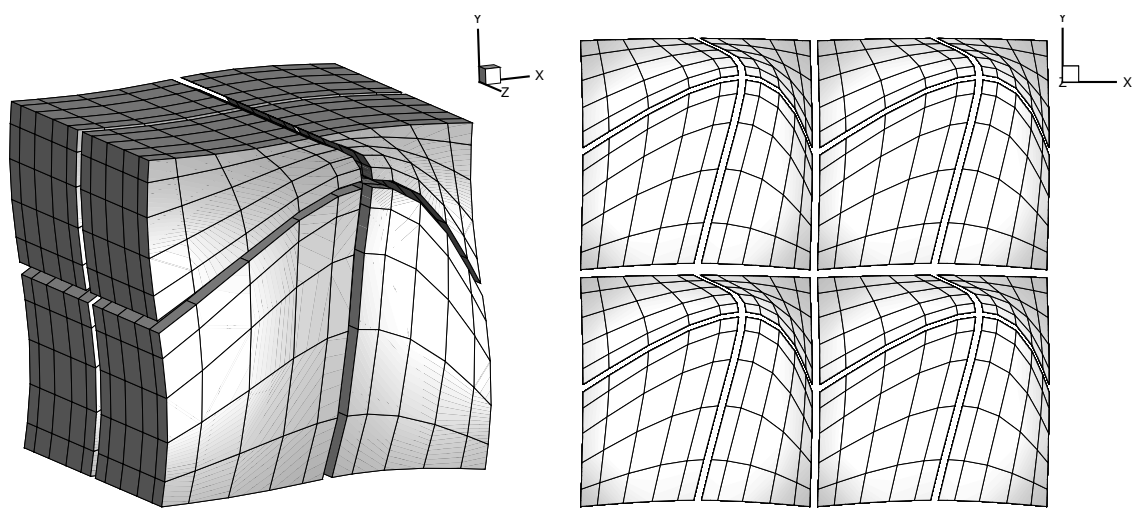

Fig. 3 Example mesh: Eight-element curvilinear mesh using six-node Legendre-Gauss distribution in each element (left). Interface slope discontinuities across periodic boundaries in the $x y$-plane (right)

of elements is constructed by subdividing the unit cube into uniform regular cubes, and the nodal distribution within each element is consistent with the chosen operator. The solutions are integrated from $t=0$ to $t=1$ using the standard explicit fourth-order Runge-Kutta time-marching method and $3 \times 10^{4}$ time steps. With this choice, the error from the time-marching method was found to be negligible relative to the error from the spatial discretization.

The accuracy, stability and conservation of the schemes are evaluated using the global norm of the discretization, $\mathbf{H}_{g}$, assembled from the local element norm matrices scaled by the appropriate transformation Jacobian on each element. For example, the accuracy of the simulation is computed relative to the exact solution of the PDE as

$$
\left\|\boldsymbol{u}_{g}-\boldsymbol{u}_{\text {exact }}\right\|_{\mathrm{H}_{g}}=\sqrt{\left(\boldsymbol{u}_{g}-\boldsymbol{u}_{\text {exact }}\right)^{T} \mathrm{H}_{g}\left(\boldsymbol{u}_{g}-\boldsymbol{u}_{\text {exact }}\right)},
$$

where $\boldsymbol{u}_{\text {exact }}$ is the vector constructed by evaluating the analytical solution at mesh nodes.

The operators chosen for this validation are based on the Legendre-Gauss (LG) and Legendre-Gauss-Lobatto (LGL) points. Both degree four and five operators are included to demonstrate the theory for both even and odd degree discretizations, as well as operators with an even or odd number of internal solution points. Results are presented using the semi-discrete equations (15) with both symmetric and upwind variations of the SATs.

\subsection{Baseline}

To demonstrate the need for the approaches presented in this article, the standard method of computing SATs is first employed. The metrics in this case are computed locally within each element with no knowledge of the element boundaries or information from adjacent elements. This approach will lead 


\begin{tabular}{|c|c|c|c|c|}
\hline & LGL - p4 & LGL - p5 & $\mathrm{LG}-\mathrm{p} 4$ & $\mathrm{LG}-\mathrm{p} 5$ \\
\hline \multicolumn{5}{|c|}{ Order of Convergence } \\
\hline Symmetric & 5.0905 & 5.6292 & 5.1930 & 5.5308 \\
\hline Upwind & 4.6615 & 5.8696 & 4.3680 & 6.3494 \\
\hline \multicolumn{5}{|c|}{ Stability - $\max \frac{d}{d t}\left(\boldsymbol{u}_{g}^{T} \mathrm{H}_{g} \boldsymbol{u}_{g}\right)$} \\
\hline Symmetric & $1.9984 \mathrm{e}-15$ & $2.2204 \mathrm{e}-15$ & $2.2204 \mathrm{e}-15$ & $1.9984 \mathrm{e}-15$ \\
\hline Upwind & $-9.1038 \mathrm{e}-15$ & 0 & $-2.4306 \mathrm{e}-09$ & $-9.3789 \mathrm{e}-11$ \\
\hline \multicolumn{5}{|c|}{ Conservation $-\max \frac{d}{d t}\left|\mathbf{1}^{\mathrm{T}} \mathrm{H}_{g} \boldsymbol{u}_{g}\right|$} \\
\hline Symmetric & $5.1174 \mathrm{e}-16$ & $6.5746 \mathrm{e}-16$ & $4.6197 \mathrm{e}-10$ & $3.294 \mathrm{e}-12$ \\
\hline Upwind & $5.2909 \mathrm{e}-16$ & $5.9848 \mathrm{e}-16$ & $4.6119 \mathrm{e}-10$ & $3.2926 \mathrm{e}-12$ \\
\hline
\end{tabular}

Table 2 Baseline: Convergence rates, as well as stability and conservation analysis for baseline simulations.

to a conservative scheme for operators whose nodal distribution includes the boundaries of the reference domain. This is because the metrics at element interfaces are computed using the same values. For operators with nodal distributions which do not include the boundaries of the reference domain, the metric values at an interface must be projected. In general, the values projected from adjacent elements to their interface will not match. This causes loss of conservation.

Considering simulations on a grid with $2^{6}$ elements, the stability and conservation of the schemes are analyzed. Table 2 shows the maximum time rate of change in the integrals of energy and of the solution. For stability the maximum time rate of change in the integral of energy should be less than or equal to zero, and for conservation the time rate of change in the integral of the solution should be machine zero. The data show that operators which include the boundary points of the reference domain are both stable and conservative, while those that do not include the boundary points of the reference domain are stable but not conservative. This highlights the need for the approaches presented in this article.

The order of convergence of the simulations is also presented in Table 2. The convergence rates are computed between two grid levels in the asymptotic region with $2^{6}$ and $2^{8}$ elements. In most cases, an order of convergence of about $p+1$ is recovered, except when using symmetric SATs and operators with an even number of nodes. In this case, a lower rate of $p$ is expected [15]. The one noticeable deviation from theory is the p4 LG simulation, which recovers an order of convergence nearly an order lower than expected. Slightly lower rates are observed from many of the $\mathrm{p} 4$ simulations using upwind SATs. In most cases, a rate more consistent with theory is observed between other grid levels.

\subsection{Low-order meshes}

One approach to obtain a conservative scheme using operators which do not include the boundary points of the reference domain is to lower the order of the computational mesh. The requirement is that the metrics can be projected 


\begin{tabular}{l||c|c||c|c} 
& LGL - p4 & LGL - p5 & LG - p4 & LG - p5 \\
\hline \multicolumn{5}{c}{ Order of Convergence } \\
\hline Symmetric & 5.2725 & 5.0490 & 5.3664 & 5.2237 \\
Upwind & 4.9965 & 5.8160 & 4.7380 & 5.9115 \\
\hline \multicolumn{5}{c}{ Stability - max $\frac{d}{d t}\left(\boldsymbol{u}_{g}^{T} \mathrm{H}_{g} \boldsymbol{u}_{g}\right)$} \\
\hline Symmetric & $1.9984 \mathrm{e}-15$ & $1.9984 \mathrm{e}-15$ & $1.9984 \mathrm{e}-15$ & $2.2204 \mathrm{e}-15$ \\
Upwind & $-2.2204 \mathrm{e}-16$ & 0 & $-2.0906 \mathrm{e}-10$ & $-1.6384 \mathrm{e}-11$ \\
\hline \multicolumn{5}{c}{ Conservation - max $\frac{d}{d t}\left|\mathbf{1}^{\mathrm{T}} \mathrm{H}_{g} \boldsymbol{u}_{g}\right|$} \\
\hline Symmetric & $5.048 \mathrm{e}-16$ & $5.8981 \mathrm{e}-16$ & $5.1348 \mathrm{e}-16$ & $5.6552 \mathrm{e}-16$ \\
Upwind & $6.297 \mathrm{e}-16$ & $5.5511 \mathrm{e}-16$ & $5.8287 \mathrm{e}-16$ & $6.2103 \mathrm{e}-16$
\end{tabular}

Table 3 B-splines of degree $\left\lfloor\frac{p}{2}\right\rfloor$ : Convergence rates, as well as stability and conservation analysis for B-spline based simulations.

exactly to the interface between adjacent elements or the boundaries of the physical domain. This implies that the metrics must be of degree less than or equal to $p$ and the mesh must be of degree $\left\lfloor\frac{p}{2}\right\rfloor$. While this approach leads to a stable and conservative method, it places a significant restriction of the mesh. In particular, it limits the accuracy with which boundaries can be represented.

There are numerous ways to construct lower-order computational meshes. In this paper, lower-order meshes are constructed from fitting a fine mesh with B-spline volumes of a prescribed polynomial degree $[34,38]$. The nodal distribution of elements in the fine mesh must contain the boundary points of the reference domain. For flexibility, accuracy and simplicity, 15 uniformly spaced nodes in each direction are used within each element to construct the fine mesh. Once each element has been fitted with a B-spline volume of a prescribed degree, the B-spline volume is evaluated at the nodal locations associated with the desired operator to generate the final mesh.

Tables 3 and 4 show the stability and conservation results using lower-order meshes constructed from B-spline fits of degree $\left\lfloor\frac{p}{2}\right\rfloor$ and $\left\lfloor\frac{p}{2}\right\rfloor+1$, respectively. The degree of the former falls within the range required for conservation, while the latter does not. As expected, this is reflected in the data: the results of the former are both stable and conservative, while the results of the latter are stable but not conservative. Even though the degree 5 LG operator appears conservative on the higher-degree mesh, conservation has dropped an order of magnitude relative to the lower-degree mesh. The loss of conservation is more apparent with the lower degree LG operator.

The tables also show the computed order of convergence from the simulations. Using the lower order B-spline fitting, the predicted order of convergence is recovered in all cases. Increasing the order of the B-spline fitting seems to lower the convergence rate of the p4 simulations using upwind SATs. Similar to the baseline simulations, more consistent convergence rates are observed between other grid levels. 


\begin{tabular}{|c|c|c|c|c|}
\hline & LGL - p4 & LGL - p5 & $\mathrm{LG}-\mathrm{p} 4$ & $\mathrm{LG}-\mathrm{p} 5$ \\
\hline \multicolumn{5}{|c|}{ Order of Convergence } \\
\hline Syn & 4.9125 & 5.2357 & 5.0822 & 5.2765 \\
\hline Upv & 4.4596 & 6.3231 & 4.3603 & 6.4464 \\
\hline \multicolumn{5}{|c|}{ Stability - $\max \frac{d}{d t}\left(\boldsymbol{u}_{g}^{T} \mathrm{H}_{g} \boldsymbol{u}_{g}\right)$} \\
\hline Syn & $2.2204 \mathrm{e}-15$ & $1.9984 \mathrm{e}-15$ & $2.2204 \mathrm{e}-15$ & $2.2204 \mathrm{e}-15$ \\
\hline Upw & $-2.2204 \mathrm{e}-16$ & $-2.2204 \mathrm{e}-16$ & $-3.8033 \mathrm{e}-10$ & $-2.7051 \mathrm{e}-11$ \\
\hline \multicolumn{5}{|c|}{ Conservation $-\max \frac{d}{d t}\left|\mathbf{1}^{\mathrm{T}} \mathrm{H}_{g} \boldsymbol{u}_{g}\right|$} \\
\hline Syl & $6.3838 \mathrm{e}-16$ & $6.3144 \mathrm{e}-16$ & $2.4945 \mathrm{e}-14$ & $1.1649 \mathrm{e}-15$ \\
\hline Upwind & $5.3603 \mathrm{e}-16$ & $5.5858 \mathrm{e}-16$ & $4.0634 \mathrm{e}-14$ & $7.1297 \mathrm{e}-16$ \\
\hline
\end{tabular}

Table 4 B-splines of degree $\left\lfloor\frac{p}{2}\right\rfloor+1$ : Convergence rates, as well as stability and conservation analysis for B-spline based simulations.

\subsection{Mortar-element approach}

The mortar-element approach removes the restriction on the order of the underlying mesh to obtain conservation. However, it does require a preprocessing step in order to compute the metrics on the interfaces between adjacent elements. This is where the mortar elements are placed. Furthermore, these metrics must satisfy the condition $1^{\mathrm{T}} c_{\kappa}=0$ (see Section 6.1 ). In this paper, we achieve this by constructing metrics that are exactly integrable by the quadrature on the mortar element. For discretizations based on LG operators this requires that the metrics on the interfaces be at most of degree $2 p+1$.

There are several ways to obtain the required metrics at the element interfaces. In this article, the B-spline fitting discussed previously is used to generate these values by analytically differentiating the equations of the Bspline volumes. A fit of degree $p$ is used, which is twice the degree required without the mortar-elements, and equal to the degree of the discretization itself. This yields metrics of degree $2 p-1$, which is lower than the LG quadrature of degree $2 p+1$ as required.

For the computations themselves, a second mesh is required on which the solution is obtained. One option is is to create a second distorted mesh built using elements with the nodal distribution associated with the desired operator. This is the how the mesh for the baseline approach is generated. Another option is to reuse the B-spline fit created to compute the metrics at the interfaces. This streamlines the workflow, and is therefore adopted in this article.

Table 5 shows the convergence, stability and conservation results of the mortar-element approach constructed from a B-spline fit of degree $p$. The results for the LG based discretization demonstrate that the addition of the mortar-elements makes the method conservative, while remaining stable. The approach also maintains these properties for operators which include the boundary points of the reference domain, as shown in the simulation of the LGL based discretization.

The orders of convergence computed from the p5 simulations using the mortar-element approach are erratic, as shown in Table 5. Rates both higher and lower than expected are recovered. Recomputing the orders of convergence 


\begin{tabular}{l||c|c||c|c} 
& LGL - p4 & LGL - p5 & LG - p4 & LG - p5 \\
\hline \multicolumn{5}{c}{ Order of Convergence } \\
\hline Symmetric & 4.9950 & $6.5735(5.1126)$ & 4.9571 & $7.4370(4.9701)$ \\
Upwind & 5.1208 & $4.7643(5.6775)$ & 4.7485 & $5.0888(5.5070)$ \\
\hline \multicolumn{5}{c}{ Stability - max $\frac{d}{d t}\left(\boldsymbol{u}_{g}^{T} \mathrm{H}_{g} \boldsymbol{u}_{g}\right)$} \\
\hline Symmetric & $2.4425 \mathrm{e}-15$ & $2.4425 \mathrm{e}-15$ & $1.9984 \mathrm{e}-15$ & $1.9984 \mathrm{e}-15$ \\
Upwind & $-1.7764 \mathrm{e}-15$ & $-1.3323 \mathrm{e}-15$ & $-6.0444 \mathrm{e}-10$ & $-2.724 \mathrm{e}-10$ \\
\hline \multicolumn{5}{c}{ Conservation - max $\frac{d}{d t}\left|\mathbf{1}^{\mathrm{T}} \mathrm{H}_{g} \boldsymbol{u}_{g}\right|$} \\
\hline Symmetric & $4.996 \mathrm{e}-16$ & $5.8981 \mathrm{e}-16$ & $5.6205 \mathrm{e}-16$ & $5.5164 \mathrm{e}-16$ \\
Upwind & $5.2909 \mathrm{e}-16$ & $5.794 \mathrm{e}-16$ & $6.1756 \mathrm{e}-16$ & $5.794 \mathrm{e}-16$
\end{tabular}

Table 5 Mortar-element approach: Convergence rates, as well as stability and conservation analysis for mortar-element approach simulations. Convergence rates shown in parenthesis are computed from coarser simulations.

between grids with $10^{4}$ and $10^{6}$ elements, yields more consistent results. This is shown in parenthesis within the table. This could be a result of the higherorder methods not being fully within the asymptotic convergence region. With the mortar-element approach, the $\mathrm{p} 4$ simulations all recover the expected order of convergence.

\subsection{Global SBP operator approach}

The global SBP operator approach adds symmetric SATs to the standard computation of the metrics and Jacobian (See A for the implementation of periodic SATs). In many ways, the global SBP operator approach is much simpler than the mortar-element approach. The modifications are only made in the computation of the metrics and Jacobian and do not change the form of the semi-discrete equations. It does not require any special knowledge of the metrics at interfaces nor additional computations to obtain such knowledge, such as the use of B-spline fitting. Furthermore, there is no optimization step required to obtain the final metrics on the interior of each element. Despite the simplicity of the global SBP approach, it is equally effective in achieving stability and conservation. This is shown in Table 6 .

The order of convergence computed from simulations using the global SBP approach are more-or-less consistent with theory, as shown in Table 6. Again, similar the the baseline simulations, slightly lower orders of convergence are recovered from the p4 simulations using upwind SATs.

\subsection{Staggered grid approach}

The staggered-grid approach requires an externally computed approximation of the Jacobian at every solution node; however, there is great flexibility in the method of constructing of the Jacobian. It only requires certain accuracy conditions in order to maintain the overall convergence rate of the discretization. Similar to the mortar-element approach, in this article we make use of 


\begin{tabular}{|c|c|c|c|c|}
\hline & LGL - p4 & LGL - p5 & LG - p4 & LG - p5 \\
\hline \multicolumn{5}{|c|}{ Order of Convergence } \\
\hline Symmetric & 5.0905 & 5.6292 & 5.1853 & 5.5688 \\
\hline Upwind & 4.6615 & 5.8695 & 4.5173 & 5.6237 \\
\hline \multicolumn{5}{|c|}{ Stability - $\max \frac{d}{d t}\left(\boldsymbol{u}_{q}^{T} \mathbf{H}_{g} \boldsymbol{u}_{g}\right)$} \\
\hline Symmetric & $1.9984 \mathrm{e}-15$ & $2.2204 \mathrm{e}-15$ & $1.7764 \mathrm{e}-15$ & $2.2204 \mathrm{e}-15$ \\
\hline Upwind & $-1.0436 \mathrm{e}-14$ & $4.4409 \mathrm{e}-16$ & $-2.8687 \mathrm{e}-09$ & $-8.5465 \mathrm{e}-11$ \\
\hline \multicolumn{5}{|c|}{ Conservation - $\max \frac{d}{d t}\left|\mathbf{1}^{\mathrm{T}} \mathrm{H}_{g} \boldsymbol{u}_{g}\right|$} \\
\hline Symmetric & $5.5685 \mathrm{e}-16$ & $6.2103 \mathrm{e}-16$ & $5.5511 \mathrm{e}-16$ & $5.5164 \mathrm{e}-16$ \\
\hline Upwind & $5.2996 \mathrm{e}-16$ & $5.7593 \mathrm{e}-16$ & $5.6552 \mathrm{e}-16$ & $6.0889 \mathrm{e}-16$ \\
\hline
\end{tabular}

Table 6 Global SBP operator: Convergence rates, as well as stability and conservation analysis for global SBP operator simulations.

\begin{tabular}{l||c|c||c|c} 
& LGL - p4 & LGL - p5 & LG - p4 & LG - p5 \\
\hline \multicolumn{5}{c}{ Order of Convergence } \\
\hline Symmetric & 4.4021 & $7.2839(4.3507)$ & 4.9539 & $7.5012(4.9720)$ \\
Upwind & 5.1054 & $4.6859(5.6683)$ & 5.1206 & $4.9818(5.4158)$ \\
\hline \multicolumn{5}{c}{ Stability - max $\frac{d}{d t}\left(\boldsymbol{u}_{g}^{T} \mathrm{H}_{g} \boldsymbol{u}_{g}\right)$} \\
\hline Symmetric & $1.9984 \mathrm{e}-15$ & $2.2204 \mathrm{e}-15$ & $1.9984 \mathrm{e}-15$ & $2.4425 \mathrm{e}-15$ \\
Upwind & $-1.7764 \mathrm{e}-15$ & $-6.6613 \mathrm{e}-16$ & $-5.7916 \mathrm{e}-10$ & $-2.5407 \mathrm{e}-10$ \\
\hline \multicolumn{5}{c}{ Conservation - max $\frac{d}{d t}\left|\mathbf{1}^{\mathrm{T}} \mathrm{H}_{g} \boldsymbol{u}_{g}\right|$} \\
\hline Symmetric & $5.5511 \mathrm{e}-16$ & $6.2797 \mathrm{e}-16$ & $6.1236 \mathrm{e}-16$ & $5.5511 \mathrm{e}-16$ \\
Upwind & $5.6725 \mathrm{e}-16$ & $5.8373 \mathrm{e}-16$ & $5.6032 \mathrm{e}-16$ & $5.7593 \mathrm{e}-16$
\end{tabular}

Table 7 Staggered grid approach: Convergence rates, as well as stability and conservation analysis for staggered grid approach simulations. Convergence rates shown in parenthesis are computed from coarser simulations.

B-splines. The value of the Jacobian is computed analytically from the equations of the B-spline volumes. Furthermore, the B-spline fit is also used to determine the flux and solution meshes in order to streamline the workflow.

The operators chosen for the flux mesh are LGL operators one degree higher than the operators applied on the solution nodes. The results of these simulations are found in Table 7. They demonstrate the staggered-grid approach also leads to stable and conservative discretizations.

The order of convergence computed from the $\mathrm{p} 4$ staggered grid simulations are reasonably consistent with theory, as shown in Table 7 . The p5 simulations, however, show considerable variation with orders of convergence both higher and lower than predicted by theory. Examining the orders of convergence computed between grids with $10^{4}$ and $10^{6}$ elements, yields slightly more consistent results, which is shown in parenthesis within the table. This is similar to the results using the mortar-element approach.

\subsection{Dense-norm SBP operators}

The dense-norm approach is very similar to the staggered-grid approach, except for the way the Jacobian is handled. In this case, the Jacobian is computed using the baseline approach on the flux grid. These values get incorporated 


\begin{tabular}{l||c|c||c|c} 
& LGL - p4 & LGL - p5 & LG - p4 & LG - p5 \\
\hline \multicolumn{5}{c}{ Order of Convergence } \\
\hline Symmetric & 4.9492 & $7.3603(4.9097)$ & 4.9544 & $7.4309(4.9123)$ \\
Upwind & 5.1559 & $4.4446(6.0717)$ & 5.0842 & $4.9839(5.3288)$ \\
\hline \multicolumn{5}{c}{ Stability - max $\frac{d}{d t}\left(\boldsymbol{u}_{g}^{T} \mathrm{H}_{g} \boldsymbol{u}_{g}\right)$} \\
\hline Symmetric & $1.9984 \mathrm{e}-15$ & $2.2204 \mathrm{e}-15$ & $1.9984 \mathrm{e}-15$ & $1.9984 \mathrm{e}-15$ \\
Upwind & $-1.7764 \mathrm{e}-15$ & $-8.8818 \mathrm{e}-16$ & $-5.6055 \mathrm{e}-10$ & $-2.2114 \mathrm{e}-10$ \\
\hline \multicolumn{5}{c}{ Conservation - max $\frac{d}{d t}\left|\mathbf{1}^{\mathrm{T}} \mathrm{H}_{g} \boldsymbol{u}_{g}\right|$} \\
\hline Symmetric & $3.2092 \mathrm{e}-15$ & $6.33 \mathrm{e}-15$ & $3.4833 \mathrm{e}-15$ & $5.5962 \mathrm{e}-15$ \\
Upwind & $3.2994 \mathrm{e}-15$ & $6.5677 \mathrm{e}-15$ & $3.1433 \mathrm{e}-15$ & $6.1062 \mathrm{e}-15$
\end{tabular}

Table 8 Dense-norm: Convergence rates, as well as stability and conservation analysis for dense-norm simulations.

on each element into both the norm matrix and interpolation/extrapolation operator from the flux mesh to the solution mesh. The results of these simulations are summarized in Table 8 and show that the dense-norm approach leads to a stable and conservative method.

The order of convergences from the dense-norm simulations are very similar to the mortar and staggered grid simulations. The $\mathrm{p} 4$ simulations are consistent with theory, whereas the higher-order p5 simulations show more variation. However, the order of convergence computed from the coarser meshes show more consistency with theory. These are shown in parenthesis within Table 8. The deviation from theory likely indicates that the higher-order simulations are not yet fully in the asymptotic region of convergence.

\section{Conclusions}

Approaches are presented that result in provably linearly stable and elementwise conservative high-order discretizations of PDEs in curvilinear coordinates for GSBP operators with either diagonal or dense norms.

A number of alternatives are presented for the construction of stable and element-wise conservative schemes using diagonal-norm GSBP operators, i.e.,

- the mortar-element approach

- the global SBP operator approach

- the staggered grid approach.

Furthermore, the staggered grid approach is extended to allow the construction of stable dense-norm operators in curvilinear coordinates. Upwind SATs for inter-element coupling are presented that add interface dissipation while maintaining the provable stability of the neutrally-stable base SBP scheme. These same SATs can be reused for the weak imposition of boundary conditions. The approaches presented in this paper can naturally be applied in the context of multidimensional SBP operators and entropy-stable discretizations of nonlinear conservation laws. Future work will concentrate on determining the advantages and disadvantages of each approach. 


$$
\begin{array}{cccccccccccc}
\mathrm{x}= & \bullet & \bullet & \bullet & \bullet & \bullet & \bullet & \bullet & \bullet & \bullet & \bullet & \bullet \\
\alpha & x_{1} & x_{2} & x_{3} & x_{4} & x_{5} & x_{6} & x_{7} & x_{8} & \beta \\
\mathrm{SAT}_{\alpha}=\mathrm{H}^{-1} \boldsymbol{t}_{\alpha}\left(\boldsymbol{t}_{\alpha}{ }^{\mathrm{T}} \boldsymbol{x}-\left(\boldsymbol{t}_{\beta}{ }^{\mathrm{T}} \boldsymbol{x}-1\right)\right) & 0.15 & 0.25 & 0.35 & 0.45 & 0.55 & 0.65 & 0.75 & 0.85 & 0.95 \\
& & & & & & & & & &
\end{array}
$$

Fig. 4 Interface SATs used for periodic faces in the computation of metric and Jacobian for the the global SBP operator approach.

\section{A Periodic boundary conditions for the global SBP operator approach}

The computation of the metrics and Jacobian involve derivatives of the physical nodal locations. The global SBP operator approach includes the use of interface SATs to couple the derivative values in adjacent elements. Unfortunately, for problems with periodic boundary conditions, the nodal locations are not necessarily periodic, even if the solution is. As a result, naively applying the SBP operator approach will not give the correct solution. In order to use the global SBP operator approach, the interface SATs for periodic boundary conditions used in the computation of the metrics and Jacobian must be modified. This requires knowledge of the domain's geometry.

For the test case used in Section 12, the size of the domain relative to any point is unity in each direction. In other words, traveling one unit along any of the coordinate directions in physical space will bring you back to the same point, even though the domain is not a unit cube. Therefore the interface SATs involving nodal locations used at periodic faces only require the addition or subtraction of a unit value. A one-dimensional example is shown in Figure 4.

\section{References}

1. Boom, P.D., Zingg, D.W.: High-order implicit time-marching methods based on generalized summation-by-parts operators. SIAM Journal on Scientific Computing 6(37), A2682-A2709 (2015)

2. Carpenter, M.H., Del Rey Fernández, D.C.: On entropy stable dicretizations using generalized summation-by-parts operators on curvilinear coordiantes. NASA Technical Report (2018)

3. Carpenter, M.H., Fisher, T.C., Nielsen, E.J., Frankel, S.H.: Entropy stable spectral collocation schemes for the Navier-Stokes equations: discontinuous interfaces. SIAM Journal on Scientific Computing 36(5), B835-B867 (2014)

4. Carpenter, M.H., Fisher, T.C., Nielsen, E.J., Parsani, M., Svärd, M., Yamaleev, N.: Entropy stable summation-by-parts formulations for computational fluid dynamics. Handbook of Numerical Analysis (17), 495-524 (2016)

5. Carpenter, M.H., Gottlieb, D., Abarbanel, S.: Time-stable boundary conditions for finite-difference schemes solving hyperbolic systems: Methodology and application to high-order compact schemes. Journal of Computational Physics 111(2), 220-236 (1994)

6. Carpenter, M.H., Nordström, J., Gottlieb, D.: A stable and conservative interface treatment of arbitrary spatial accuracy. Journal of Computational Physics 148(2), 341-365 (1999) 
7. Carpenter, M.H., Nordström, J., Gottlieb, D.: Revisiting and extending interface penalties for multi-domain summation-by-parts operators. Journal of Scientific Computing 45(1-3), 118-150 (2010)

8. Carpenter, M.H., Parsani, M., Fisher, T.C., Nielsen, E.J.: Towards an entropy stable spectral element framework for computational fluid dynamics. In: 54th AIAA Aerospace Sciences Meeting, AIAA 2016-1058. American Institute of Aeronautics and Astronautics (AIAA) (2016)

9. Chan, J.: Weight-adjusted discontinuous Galerkin methods: Matrix-valued weights and elastic wave propagation in heterogeneous media. International Journal for Numerical Methods in Engineering 113(12), 1779-1809 (2017)

10. Chan, J.: On discretely entropy conservative and entropy stable discontinuous Galerkin methods. Journal of Computational Physics 362, 346 - 374 (2018)

11. Chan, J., Del Rey Fernández, D.C., Carpenter, M.H.: Efficient entropy stable Gauss collocation methods. (Submitted to SIAM Journal on Scientific Computing) (2018)

12. Chan, J., Hewett, R.J., Warburton, T.: Weight-adjusted discontinuous Galerkin methods: wave propogation in hetrogeneous media. SIAM Journal on Scientific Computing 39(6), A2935-A2961 (2017)

13. Chan, J., Hewett, R.J., Warburton, T.: Weight-adjusted discontinuous Galerkin methods: curvilinear meshes. arXiv:1608.03836v1 [math.NA] (2018)

14. Chen, T., Shu, C.W.: Entropy stable high order discontinuous Galerkin methods with suitable quadrature rules for hyperbolic conservation laws. Journal of Computational Physics 345, 427 - 461 (2017)

15. Cheng, Y., Chou, C.S., Li, F., Xing, Y.: L2 stable discontinuous Galerkin methods for one-dimensional two-way wave equations. Math. Comput. 86, 121-155 (2017)

16. Crean, J., Hicken, J.E., Del Rey Fernández, D.C., Zingg, D.W., Carpenter, M.H.: Entropy-stable summation-by-parts discretization of the Euler equations on general curved elements. Journal of Computational Physics 356, $410-438$ (2018)

17. Del Rey Fernández, D.C., Boom, P.D., Zingg, D.W.: A generalized framework for nodal first derivative summation-by-parts operators. Journal of Computational Physics 266(1), 214-239 (2014)

18. Del Rey Fernández, D.C., Carpenter, M.H., Friedrich, L., Winters, A.R., Gassner, G.J., Dalcin, L., Parsani, M.: Entropy stable non-conforming discretizations with the summation-by-parts property for curvilinear coordinates. NASA Technical Report, 2018 (2018)

19. Del Rey Fernández, D.C., Crean, J., Carpenter, M.H., Hicken, J.E.: Staggered-grid entropy-stable multidimensional summation-by-parts discretizations on curvilinear coordinates. (Accepted: Journal of Scientific Computing) (2019)

20. Del Rey Fernández, D.C., Hicken, J.E., Zingg, D.W.: Review of summation-by-parts operators with simultaneous approximation terms for the numerical solution of partial differential equations. Computers \& Fluids 95(22), 171-196 (2014)

21. Del Rey Fernández, D.C., Hicken, J.E., Zingg, D.W.: Simultaneous approximation terms for multidimensional summation-by-parts operators. Journal of Scientific Computing 1(75) (2018)

22. Deng, X., Mao, M., Tu, G., Liu, H., Zhang, H.: Geometric conservation law and applications to high-order finite difference schemes with stationary grids. Journal of Computational Physics 230(4), 1100-1115 (2011)

23. Deng, X., Min, Y., Mao, M., Liu, H., Tu, G., Zhang, H.: Further studies on geometric conservation law and applications to high-order finite difference schemes with stationary grids. Journal of Computational Physics 239, 90-111 (2013)

24. Derigs, D., Winters, A.R., J.Gassner, G., Walch, S.: A novel averaging technique for discrete entropy-stable dissipation operators for ideal mhd. Journal of Computational Physics 330(1), 624-632 (2017)

25. Fisher, T.C.: High-order $l^{2}$ stable multi-domain finite difference method for compressible flows. Ph.D. thesis, Purdue University (2012)

26. Fisher, T.C., Carpenter, M.H.: High-order entropy stable finite difference schemes for nonlinear conservation laws: Finite domains. Journal of Computational Physics 252(1), $518-557$ (2013) 
27. Fisher, T.C., Carpenter, M.H.: High-order entropy stable finite difference schemes for nonlinear conservation laws: Finite domains. Journal of Computational Physics 252 , 518-557 (2013)

28. Fisher, T.C., Carpenter, M.H., Nordström, J., Yamaleev, N.K.: Discretely conservative finite-difference formulations for nonlinear conservation laws in split form: Theory and boundary conditions. Journal of Computational Physics 234(1), 353-375 (2013)

29. Friedrich, L., Del Rey Fernández, D.C., Winters, A.R., Gassner, G.J., Zingg, D.W. Hicken, J.E.: Conservative and stable degree preserving SBP operators for nonconforming meshes. Journal of Scientific Computing 75(2), 657-686 (2018)

30. Friedrich, L., Winters, A.R., Del Rey Fernández, D.C., Gassner, G.J., Parsani, M. Carpenter, M.H.: An entropy stable $h / p$ non-conforming discontinuous Galerkin method with the summation-by-parts property. Journal of Scientific Computing pp. 1-37 (2018)

31. Gassner, G.J.: A skew-symmetric discontinuous Galerkin spectral element discretization and its relation to SBP-SAT finite difference methods. SIAM Journal on Scientific Computing 35(3), A1233-A1253 (2013)

32. Gassner, G.J., Winters, A.R., Kopriva, D.A.: A well balanced and entropy conservative discontinuous Galerkin spectral element method for the shallow water equations. Applied Mathematics and Computation 272(2), 291-308 (2016)

33. Hicken, J.E., Del Rey Fernández, D.C., Zingg, D.W.: Multidimensional summation-bypart operators: General theory and application to simplex elements. SIAM Journal on Scientific Computing 4(38) (2016)

34. Hicken, J.E., Zingg, D.W.: Aerodynamic Optimization Algorithm with Integrated Geometry Parameterization and Mesh Movement. AIAA Journal 48(2), 400-413 (2010)

35. Lax, P.D., Wendroff, B.: Systems of conservation laws. Communications on pure and applied mathematics 13, 217-237 (1960)

36. Nordström, J., Carpenter, M.H.: Boundary and interface conditions for high-order finitedifference methods applied to the Euler and Navier-Stokes equations. Journal of Computational Physics 148(2), 621-645 (1999)

37. Nordström, J., Carpenter, M.H.: High-order finite-difference methods, multidimensional linear problems, and curvilinear coordinates. Journal of Computational Physics 173(1), 149-174 (2001)

38. Osusky, L.M.: A numerical methodology for aerodynamic shape optimization in turbulent flow enabling large geometric variation. Ph.D. thesis, University of Toronto (2014)

39. Parsani, M., Carpenter, M.H., Fisher, T.C., Nielsen, E.J.: Entropy stable staggered grid discontinuous spectral collocation methods of any order for the compressible NavierStokes equations. SIAM Journal on Scientific Computing 38(5), A3129-A3162 (2016)

40. Parsani, M., Carpenter, M.H., Nielsen, E.J.: Entropy stable wall boundary conditions for the three-dimensional compressible Navier-Stokes equations. Journal of Computational Physics 292(1), 88-113 (2015)

41. Ranocha, H., Glaubitz, J., Öffner, P., Sonar, T.: Stability of artificial dissipation and modal filtering for flux reconstruction schemes using summation-by-parts operators. Applied Numerical Mathematics 128, 1-23 (2018)

42. Ranocha, H., Öffner, P., Sonar, T.: Summation-by-parts operators for correction procedure via reconstruction. Journal of Computational Physics 311(15), 299-328 (2016)

43. Ranocha, H., Öffner, P., Sonar, T.: Extended skew-symmetric form for summation-byparts operators and varying jacobians. Journal of Computational Physics 342(C), 13-28 (2017)

44. Shi, C., Shu, C.W.: On local conservation of numerical methods for conservation laws. Computers \& Fluids 169(4), 3-9 (2018)

45. Svärd, M.: On coordinate transformations for summation-by-parts operators. Journal of Scientific Computing 20(1), 29-42 (2004)

46. Svärd, M., Nordström, J.: Review of summation-by-parts schemes for initial-boundaryvalue-problems. Journal of Computational Physics 268(1), 17-38 (2014)

47. Thomas, D., Lombard, C.K.: Geometric conservation law and its application to flow computations on moving grids. AIAA Journal 17(10), 1030-1037 (1979)

48. Vinokur, M., Yee, H.C.: Extension of efficient low dissipation high order schemes for 3-D curvilinear moving grids. In: D.A. Caughey, M. Hafez (eds.) Frontiers of Computational Fluid Dynamics, pp. 129-164. World Scientific Publishing Company (2002) 
49. Wintermeyer, N., Winters, A.R., Gassner, G.J., Kopriva, D.A.: An entropy stable nodal discontinuous Galerkin method for the two dimensional shallow water equations on unstructured curvilinear meshes with discontinuous bathymetry. Journal of Computational Physics 340(1), 200-242 (2017)

50. Winters, A.R., Derigs, D., Gassner, G.J., Walch, S.: Uniquely defined entropy stable matrix dissipation operator for high mach number ideal MHD and compressible Euler simulations. Journal of Computational Physics 332(1), 274-289 (2017)

51. Winters, A.R., Gassner, G.J.: Affordable, entropy conserving and entropy stable flux functions for the ideal mhd equations. Journal of Computational Physics 304(1), 72-108 (2016)

52. Winters, A.R., J.Gassner, G.: A comparison of two entropy stable discontinuous Galerking spectral element approximations to the shallow water equations with non-constant topography. Journal of Computational Physics 301(1), 357-376 (2015)

53. Yamaleev, N.K., Carpenter, M.H.: A family of fourth-order entropy stable nonoscillatory spectral collocation schemes for the 1-d Navier-Stokes equations. Journal of Computational Physics 331, 90-107 (2017) 